\title{
Tuning crystal ordering, electronic structure and morphology in organic semiconductors: Tetrathiafulvalenes as a model case.
}

\author{
Raphael Pfattner, Stefan T. Bromley, Concepció Rovira and Marta Mas-Torrent*
}

\section{[*] Dr. M. Mas-Torrent, Prof. C. Rovira, Dr. R. Pfattner}

Institut de Ciència de Materials de Barcelona (ICMAB-CSIC), and Networking Research

Center on Bioengineering, Biomaterials and Nanomedicine (CIBER-BBN), Campus UAB, 08193 Bellaterra (Spain)

E-mail: mmas@icmab.es

Prof. Dr. Stefan T. Bromley,

Departament de Química Física and Institut de Química Teòrica i Computacional (IQTCUB), Universitat de Barcelona, 08028 Barcelona, and Institució Catalana de Recerca i Estudis Avançats (ICREA), 08010, Barcelona, Spain

Keywords: Tetrathiafulvalene, organic field-effect transistor, organic-semiconductor, small molecule, structure-performance correlation

Tetrathiafulvalenes (TTFs) are an appealing class of organic small molecules giving rise to some of the highest performing active materials reported for organic field effect transistors (OFETs). Because they can be easily chemically modified, TTF-derivatives are ideal candidates to perform molecule-property correlation studies and, especially, to elucidate the impact of molecular and crystal engineering on device performance. A brief introduction into the state-of-the-art of the field-effect mobility values achieved with TTF derivatives employing different fabrication techniques is provided. Following, structure-performance relationships are discussed, including polymorphism, a phenomenon which is crucial to control for ensuring device reproducibility. It is also shown that chemical modification of TTFs has a strong influence on the electronic structure of these materials, affecting their stability as well as the nature of the charge carriers, leading to devices with p-channel, nchannel or even ambipolar behaviour. TTFs have also shown promise in other applications, such as phototransistors, sensors, or as dopants or components of organic metal charge transfer salts used as source-drain contacts. Overall, TTFs are appealing building blocks in 
organic electronics, not only because they can be tailor designed to perform fundamental studies, but also because they offer a wide spectrum of potential applications.

\section{Introduction}

The first idea of an insulated-gate field-effect transistor was presented in the 1926's by Lilienfeld et al., ${ }^{[1]}$ but it was only in 1947 that the point-contact-transistor was demonstrated by Brattain, Bardeen and Shockley. ${ }^{[2]}$ Germanium, which was the first used material in diodes and transistors, was soon replaced by silicon. The invention of the integrated circuit (Metal Oxide Semiconductor Field-Effect Transistor (MOSFET)) in 1960 was another important milestone towards the information age as since then the microelectronic industry grew rapidly. $^{[3,4]}$ In the 1980's Organic Field-Effect Transistors (OFETs) started to appear ${ }^{[5,6]}$ raising a huge interest from a fundamental point of view as well as from a technological perspective. The main advantage of OFETs with respect to inorganic-based electronics is that the processing characteristics of organic semiconductors are potentially compatible with electronic applications where low-cost, large area coverage and structural flexibility are required. Further, the possibility to design and synthesize organic semiconductors for specific purposes or for tuning the device properties represents a clear added value. In fact, the first organic-based devices are already emerging in the market. Among them we find flexible, glass-free electronic papers, ${ }^{[7]}$ curved smart phones, ${ }^{[8]}$ or OLED based TV screens. ${ }^{[9]}$

The basic configuration of an OFET comprises three electrodes (source, drain and gate), a dielectric layer, and an active semiconductor film in close contact with the dielectric. In sharp contrast to inorganic field-effect transistors, OFETs are generally driven in the accumulation regime, which means that charge carries are accumulated at the interface between the organic semiconductor and the dielectric when an electric field is applied by the gate voltage $\left(\mathrm{V}_{\mathrm{G}}\right)$. Considering this, it is important to keep in mind thus that the charge carrier transport in an 
OFET is known to take place in the first few molecular monolayers of the organic semiconductor. ${ }^{[10]}$ Other than that, in principle, the organic field-effect transistor behaves similarly to its inorganic counterpart and, in fact, classical MOSFET theory is often applied to describe its electrical characteristics.

Organic semiconducting materials can be classified into two major groups: ${ }^{[11]}$ i) conjugated polymers which can be either of amorphous (e.g. polytriarylamines (PTAA)), or polycrystalline nature (e.g. polythiophenes), and ii) small conjugated molecules (e.g. pentacene, oligothiophene, tetrathiafulvalene, perylene diimide). Typically, polymers can be easily processed from solution, but molecular disorder limit the charge transport. ${ }^{[12]}$ On the other hand, small molecules form more crystalline structures, but, since they are more difficult to process from solution, they are often deposited employing vacuum deposition techniques. To circumvent this issue, solubilising groups have been incorporated into small molecule semiconductors in an attempt to impart solubility to the constituent molecules without disturbing the formation of suitable molecular packings for charge transport.

From a fundamental point of view, the charge transport properties of an organic molecule are strongly related to the Highest Occupied Molecular Orbital (HOMO) and the Lowest Unoccupied Molecular Orbital (LUMO), schematically shown in Figure 1. Extending this view towards an organic solid it becomes very clear that the relative position of the HOMO LUMO as well as the intermolecular interactions reflected in HOMO/HOMO, in case of pchannel materials, and LUMO/LUMO overlap, in n-channel materials, play a crucial role. Usually the more simplified scheme in Figure 1 is successfully used to describe the electrical characteristics of different devices. 


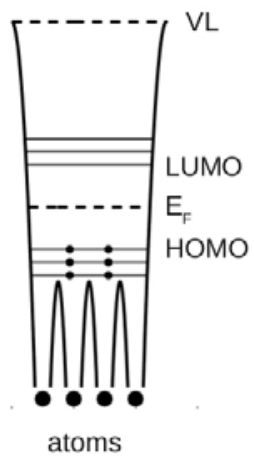

a) molecule

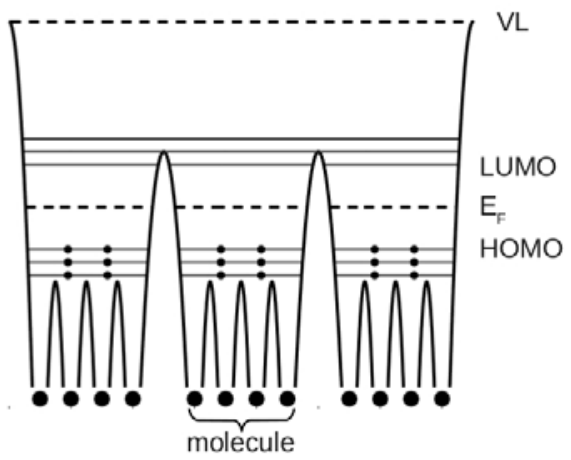

b) organic solid

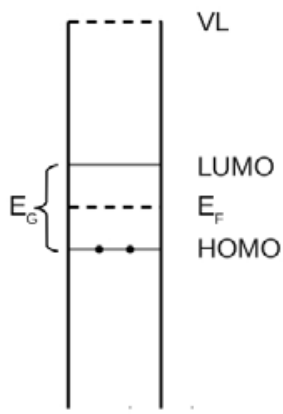

c) simplified model

Figure 1. Schematic representation of energy levels important to consider for charge transport in organic solids. Vacuum level (VL), Highest Occupied Molecular Orbital (HOMO), Lowest Unoccupied Molecular Orbital (LUMO), Fermi Energy $\left(\mathrm{E}_{\mathrm{F}}\right)$ and Energy Gap $\left(E_{G}\right)$ of a) isolated organic molecule, b) organic solid and c) simplified model.

The key parameter in OFETs is the field-effect mobility $\left(\mu_{\mathrm{FE}}\right)$. In an ideal device this value is close to the intrinsic mobility $(\mu)$ of the active material which is mainly determined by the $\pi-\pi$ intermolecular interactions and therefore, it is closely related to the crystal packing. However, in reality there are many other factors affecting the measured mobility. Contact resistance can appear if the HOMO, for a p-channel material, or the LUMO, for an n-channel material, is not well-aligned with the metal work function. Besides $\mu_{\mathrm{FE}}$, on-off current ratio is an important device parameter in OFETs and values exceeding $10^{6}$ have been reported in literature. ${ }^{[13]}$

Further, many factors, such as the degree of crystallinity, the thin film morphology (i.e. grain boundaries) or the presence of defects at the dielectric/semiconductor interface can play a crucial role in the final device performance. The fundamental material characteristics of organic semiconductors are most clearly measured in single-crystals. Indeed, single crystal OFETs ${ }^{[14]}$ can be regarded as model systems in order to carry out correlation studies between 
crystal structure and device performance since in these devices issues originating from grain boundaries, film morphology and crystallinity can be minimized.

Among the different families of small molecules organic semiconductors, tetrathiafulvalenes (TTFs) have been extensively studied in the last few years. After the discovery of the first organic metal tetrathiafulvalene-tetracyanoquinodimethane (TTF-TCNQ) back in $1973,{ }^{[15,16]}$ a vast number of metallic and even superconducting charge transfer salts based on TTFs were reported. ${ }^{[17]}$ However, the application of TTFs as organic semiconductors was only realised more than 25 years later. ${ }^{[18]}$ The generally good solubility of this material in common organic solvents and its well-known chemistry, permits the design and synthesis of new materials almost at will. This, together with the good field effect mobility reported for some TTF derivatives-based OFETs, makes this molecule an ideal system to perform correlation studies of the device characteristics with respect to structural and morphological related aspects. In addition, by proper design and synthesis the electronic or crystal structure can also be significantly tuned. This feature paper aims at showing how different molecular, material and device parameters can affect the final OFET performance. This is elucidated considering recent work performed in the fabrication of TTF OFETs, which can be considered as model systems.

\section{Tetrathiafulvalene as organic semiconductors}

Tetrathiafulvalene (TTF, Figure 2, top) is a rich electron donor organosulfur compound. Oxidation of the TTF ring system leads to the cation radical and dication species sequentially and reversibly within a very accessible potential window. ${ }^{[19,20]}$ Each dithiolylidene ring of the neutral TTF has $7 \pi$ electrons: 2 for each sulphur atom and 1 for each $\mathrm{sp}^{2}$ carbon atom. Removing one of these electrons (i.e. oxidation) from the neutral TTF leads to the formation of a radical cation where one of the two rings becomes aromatic with a delocalization of the 
remaining electron system in the fulvalene moiety. Similarly, when a second electron is removed from the TTF rings, the radical dication consisting of $6 \pi$ electrons per ring is formed. Thus, the oxidised TTF species are thermodynamically very stable. By adding electron withdrawing or electron donating groups to the TTF core the redox potentials, and thus, the HOMO and LUMO levels, can be easily tuned. This property makes them very versatile for fundamental studies and the ideal starting material for tailor-made organic semiconductors. $^{[21,22]}$

From a supramolecular point of view, TTFs show a high tendency to form ordered stacks or two-dimensional sheets, which are stabilized by both intermolecular $\pi-\pi$ and $S \cdots S$ interactions (Figure 2, bottom).

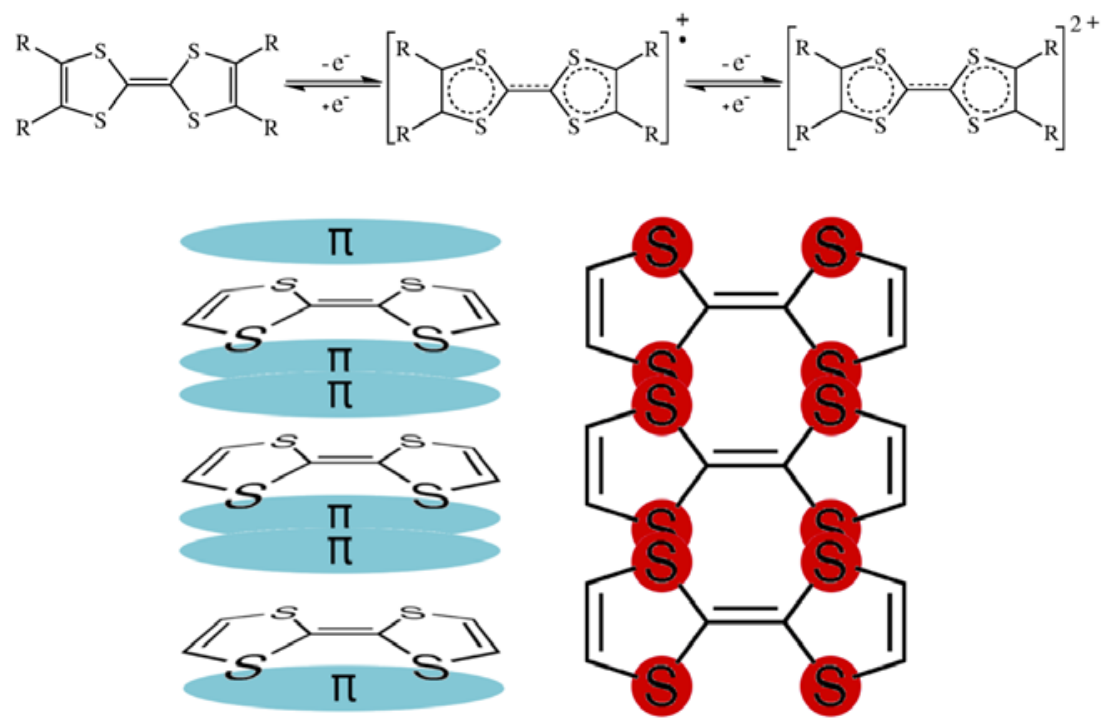

Figure 2. Tetrathiafulvalene derivatives (TTF). Top. Oxidation processes of tetrathiafulvalene (TTF) to radical cation and dication species. Bottom. Schematic representation of the main intermolecular interactions, $\pi-\pi$ orbital overlap and $S \cdots S$ interactions, that govern the supramolecular organization of TTF crystals. $\pi$ - orbitals above and below the molecular plane and sulfur (S) atoms are shown in light blue and red, respectively.

TTF-derivatives are generally soluble in conventional organic solvents and stable, avoiding strong acid conditions or strong oxidants, to a large number of synthetic transformations which are important features for the design of customized active materials in potential 
devices. ${ }^{[18]}$ The solubility can be further increased by adding functional groups such as alkyl chains making them suitable for solution processing at higher concentrations. ${ }^{[23,24]}$

\section{State-of-the-art of TTF OFETs}

TTF OFETs have been studied using a wide range of material deposition methods and achieving performance matching those reported with benchmark semiconductors. ${ }^{[25]}$ Here we will discuss some of the best performing devices, but for a detailed description of the performance of TTF OFETs we refer the reader to reference. ${ }^{[21]}$

A suitable technique to prepare homogenous thin-films in a controlled fashion is thermal evaporation. Since organic materials typically sublimate at low temperatures a crucible heated by an electrical current in high vacuum permits the deposition of thin-films. Table 1 gives a summary of some of the best mobility values reported for TTF derivatives prepared from vacuum deposition. As it can be seen, in thermally evaporated thin film OFETs mobility values above $0.1 \mathrm{~cm}^{2} / \mathrm{Vs}$ have been obtained with different derivatives. ${ }^{[26-31]}$ It should be considered that the evaporation parameters such as vacuum pressure, substrate pre-treatment, substrate temperature and deposition rate can lead to considerable variations in thin-film morphology for a specific active material. ${ }^{[32,33]}$ For instance, in di(biphenyl)-tetrathiafulvalene (DBP-TTF) evaporated films a larger grain size, confirmed by X-ray analysis, has been found for films evaporated at $80^{\circ} \mathrm{C}$ compared to films evaporated at room temperature. ${ }^{[29,34]}$ Additionally, substrate pre-treatment by the application of a monolayer such as, HMDS (hexamethyldisilazane), ${ }^{[28,35]}$ OTS (octadecyltrichlorosilane) ${ }^{[36,37]}$ or the use of polymeric substrates such as PC (polycarbonate), PMMA (poly(methyl methacrylate)), PS

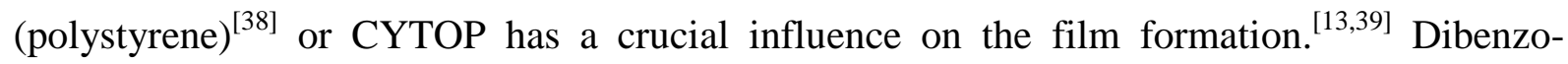
tetrathiafulvalene (DB-TTF) has been the most studied TTF derivative due to its facile 
synthesis and $\pi$-extended nature. However, reported DB-TTF evaporated thin films display a wide range of mobility values. ${ }^{[33,40-42]}$ Polycrystalline-film morphologies of DB-TTF were investigated on $\mathrm{Si} / \mathrm{SiO}_{2}$ surfaces treated with HMDS following a variety of methods for the oxide functionalisation. Depending on such pre-treatment method, the hydrophobocity of the surfaces was modified, which, in turn affected the lateral grain sizes of the evaporated DBTTF film from 0.2 up to $20 \mu \mathrm{m}$. When the surfaces were more hydrophobic, the surface diffusion of DB-TTF molecules was favoured promoting the two-dimensional growth as well as the coalescence of the grains. Accordingly, the OFET performances ranged from 0.35 to $0.55 \mathrm{~cm}^{2} / \mathrm{Vs}$, being the highest values achieved when the grain sizes were maximised. ${ }^{[35]}$ This clearly reflects the importance of the dielectric surface on the device performance. It should be also mentioned that DB-TTF thin film OFETs show typically high positive threshold voltage values and are not very stable in air. However, it was reported that the functionalisation of DB-TTF with tert-butyl groups significantly improved the ambient stability of the devices, which was tentatively attributed to a passivation effect of the bulky tert-butyl groups that suppressed the generation of traps at the interface. ${ }^{[40]}$ Thermally evaporated thin-film transistors based on octamethylene-tetrathiafulvalene (OM-TTF) modified with $t$-butyl showed higher performance compared to the parent OM-TTF but, here the effect was ascribed to the promotion of a standing molecular arrangement. ${ }^{[43]}$ High hole mobilities have been reported for other DB-TTF analogues such as those with fused bisimides and benzothiadiazole, giving values of up to 0.4 and $0.73 \mathrm{~cm}^{2} / \mathrm{Vs}$, respectively, for thin films evaporated on top of OTS treated $\mathrm{SiO}_{2}$ dielectric at moderate substrate temperatures $\left(30{ }^{\circ} \mathrm{C}\right) .{ }^{[40,43-46]}$ Cyano-substituted TTF has shown to form good evaporated thin films on a $\mathrm{SiO}_{2}$ substrate on account of the good affinity of the polar cyano groups to the inorganic substrate, exhibiting a mobility of up to $0.02 \mathrm{~cm}^{2} / \mathrm{Vs}^{[27]}$

The highest OFET mobility in an evaporated thin-film TTF-derivative has been found for hexamethylene-tetrathiafulvalene (HM-TTF). Thin films of this material were vacuum 
deposited on $\mathrm{Si} / \mathrm{SiO}_{2}$ substrates coated with a thin film of a series of different polymers. All the films produced exhibited similar X-ray diffraction patterns, but by AFM and X-ray analysis it was concluded that the grains in the films on PS were highly oriented with their $b$ axes perpendicular to the surface, while they were not oriented on the other surfaces. Interestingly, the devices on PS exhibited a field effect mobility as high as $6.9 \mathrm{~cm}^{2} / \mathrm{Vs}$, again elucidating the importance on the organic semiconductor-dielectric interface. ${ }^{[13]} \mathrm{HM}-\mathrm{TTF}$ substituted with bulky alkyl groups have also shown high mobilities. ${ }^{[47]}$

Another important parameter is the base pressure employed during thermal evaporation of a TTF material, which can have a considerable impact on the film quality. In contrast to what is expected, in the case of tetramethyl-tetrathiafulvalene (TM-TTF), higher performance was achieved when the thin films were prepared under low vacuum conditions achieving a maximum mobility of $0.55 \mathrm{~cm}^{2} / \mathrm{Vs} .{ }^{[39]}$ However, the highest reported mobility value for this material was realized in TCNQ/TM-TTF self-contacted transistors with a value of up to 0.68 $\mathrm{cm}^{2} /$ Vs (see Source-Drain contacts Section). ${ }^{[30]}$

A widely used technique to grow high quality single crystals is vapour transport which is often also used for efficiently purifying organic materials. An inert gas is typically used to transport the sublimated organic material from the heat source along a glass tube with a controlled temperature gradient. The organic material condensates at the other end of the glass tube at lower temperature. Despite that this technique has been extensively used for growing pure crystals of benchmark semiconductors such as acene or thiophene derivatives, there are not many reports on TTFs. For single crystals of tetramethyl-tetraselenefulvalene (TM-TSF) fabricated using this technique a moblilty of about $0.2 \mathrm{~cm}^{2} / \mathrm{Vs}$ was extracted at room temperature using a polyethylene terephthalate (PET) film as a dielectric. ${ }^{[48]}$ A thermally activated charge transport, independent of the source/drain materials used, i.e. Au and graphite paste, was observed in these devices. ${ }^{[48]}$ The intrinsic charge transport properties of single crystals of the same material have also been extracted by using an air gap as gate 
dielectric exhibiting mobility values in the linear regime of around $4 \mathrm{~cm}^{2} / \mathrm{Vs}$ at room temperature. ${ }^{[49]}$ In these samples, the mobility increased for a range of temperatures following a metal-like behaviour reaching a maximum value, after which $\mu$ decreased with further lowering the temperature. ${ }^{[49]}$ Remarkably, OFETs based on single crystals of HM-TTF obtained by vapour transport gave a mobility of $11.2 \mathrm{~cm}^{2} / \mathrm{Vs}$ when the organic metal TTFTCNQ was used as source-drain contacts. ${ }^{[50]}$ This mobility value is among the highest reported for organic semiconductors.

Table 1. OFET mobility values reported in thermally evaporated thin-films and single crystals grown by physical vapour deposition of TTF derivatives.

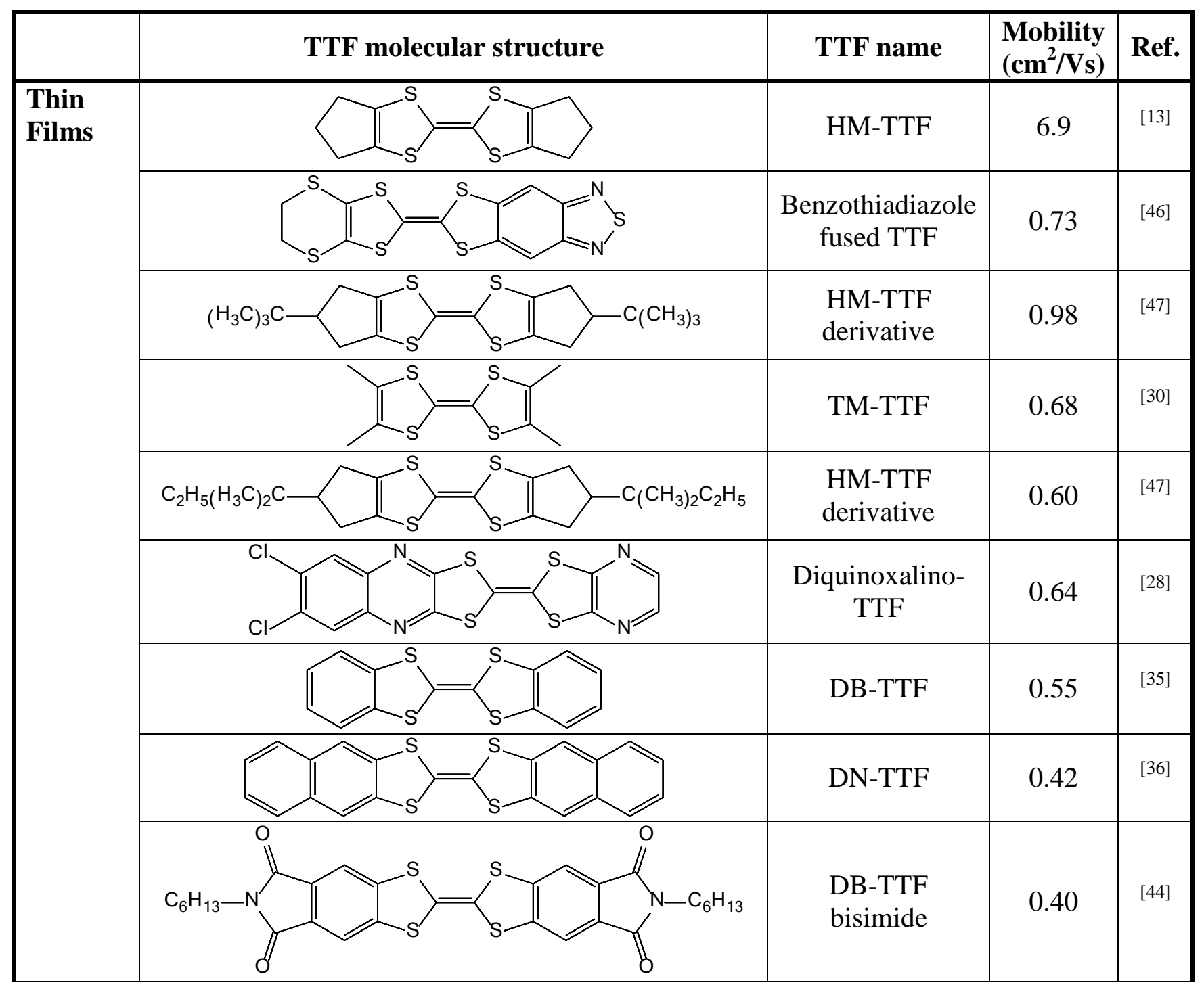




\begin{tabular}{|l|l|l|l|l|}
\hline \multicolumn{1}{|c|}{ Single } \\
Crystals
\end{tabular}

As previously mentioned, one of the most appealing characteristics of TTF derivatives is that they are typically soluble in common organic solvents. Thus, solution processed devices have been reported employing TTFs (Table 2). An attractive technique for the preparation of thin films using soluble materials is the so-called Zone Casting technique. ${ }^{[51,52]}$ Controlled supply of a TTF solution in a high boiling point solvent at controlled temperature to a substrate with controlled temperature using a constant flow rate and movement of the substrate permits the crystallization of highly ordered thin-films. This technique was applied with tetrakis-(alkyl)tetrathiafulvalenes (TTF-4SC ) and DT-TTF, which showed a mobility of up to 0.25 $\mathrm{cm}^{2} / \mathrm{Vs}^{[53]}$ and $0.17 \mathrm{~cm}^{2} / \mathrm{Vs},{ }^{[54]}$ respectively.

Spin-coating is another solution based technique that has been employed to process TTFs for preparing homogenous thin films. ${ }^{[23,55-58]}$ For this purpose, also TTFs bearing alkyl chains have been employed in order to increase the solubility to some extend and to realise more viscous solutions with higher TTF concentration. Thus, N-alkyl-substituted bis(pyrrolo[3,4d])tetrathiafulvalenes (PyTTs) with mobilities of up to $0.013 \mathrm{~cm}^{2} / \mathrm{Vs}$ have been achieved with films prepared by spin-coating. ${ }^{[23]}$ Furthermore, the modification of DB-TTF with alkylchains promotes the molecules to stand perpendicular to the substrate and the deposited films show similar performance as those found in thermally evaporated films of DB-TTF. ${ }^{[58]}$ Spin 
coated films of linear benzene-fused bis-tetrathiafulvalenes (benzene-bisTTF) exhibited a mobility of up to $0.02 \mathrm{~cm}^{2} / \mathrm{Vs}{ }^{[24,55,56]}$ One of the highest field-effect moblitities in OFETs prepared by spin-coating has been reported for a bis-tetrathiafulvalene fused-naphthalene diimide (TTF-NDI-TTF) using high concentrations of $10 \mathrm{mg} / \mathrm{ml}$ in chloroform. The resulting thin films after vacuum annealing at $160{ }^{\circ} \mathrm{C}$ revealed a mobility of $0.31 \mathrm{~cm}^{2} / \mathrm{Vs}{ }^{[56]}$

Previously, it was mentioned that not much work has been focused on the fabrication of TTF single crystal OFETs prepared from the vacuum phase. However, due to their high tendency to crystallise from solution, substantial efforts have been devoted to the preparation of solution processed TTF single crystal OFETs, achieving very high OFET mobility values comparable to the ones reported for vapour grown organic semiconductors. The most extended technique that has been used to grow crystals is by drop casting a solution of the TTF in a high boiling point solvent on the top of the substrate and allowing the solvent to evaporate slowly. ${ }^{[38,59-63]}$ In this approach, the substrate might contain pre-fabricated electrodes or otherwise the crystals formed can also be manually connected with a conducting graphite paste. Single crystals grown by drop casting using a variety of TTF derivatives have resulted in devices reaching mobilities of up to $6.2 \mathrm{~cm}^{2} / \mathrm{Vs}$ for the $\alpha$ polymorph of DT$\mathrm{TTF}^{[61,64-66]}$

An interesting study has been recently reported by Liu et al. where large oriented TTF single crystal microwire arrays were grown on top of $\mathrm{Si} / \mathrm{SiO}_{2}$ substrates in the saturated solvent atmosphere using an optimised concentration. ${ }^{[67]}$ This group further extended this methodology with DB-TTF casting multiple droplets of the semiconductor solution in dichloromethane at predetermined regions on a substrate and allowing them to evaporate slowly. The fusion of two adjacent droplets provided a region with a lower evaporation rate resulting in well-aligned microwires located at a definite position. ${ }^{[68]}$

HM-TTF has also given the highest mobility in solution prepared single crystals, as in the case of evaporated thin films or single crystals grown by vapour transport, giving devices with 
a mobility of up to $10.4 \mathrm{~cm}^{2} / \mathrm{Vs}$ when organic contacts were used. ${ }^{[50]}$ Here, the crystals were obtained by the slow cooling and evaporation of a solution of the TTF derivative over several weeks at room temperature.

Another appealing crystallisation strategy was reported for benzothiadiazoletetrathiafulvalene (BT-TTF). This material was dissolved in a mixture of high boiling point solvents and then methanol was added forming a suspension of crystals. Such suspension was then drop casted on the device that gave mobilities of up to $0.36 \mathrm{~cm}^{2} / \mathrm{Vs}{ }^{[69]}$

Table 2. OFET mobility values reported in solution processed thin-film and single crystals of TTFs.

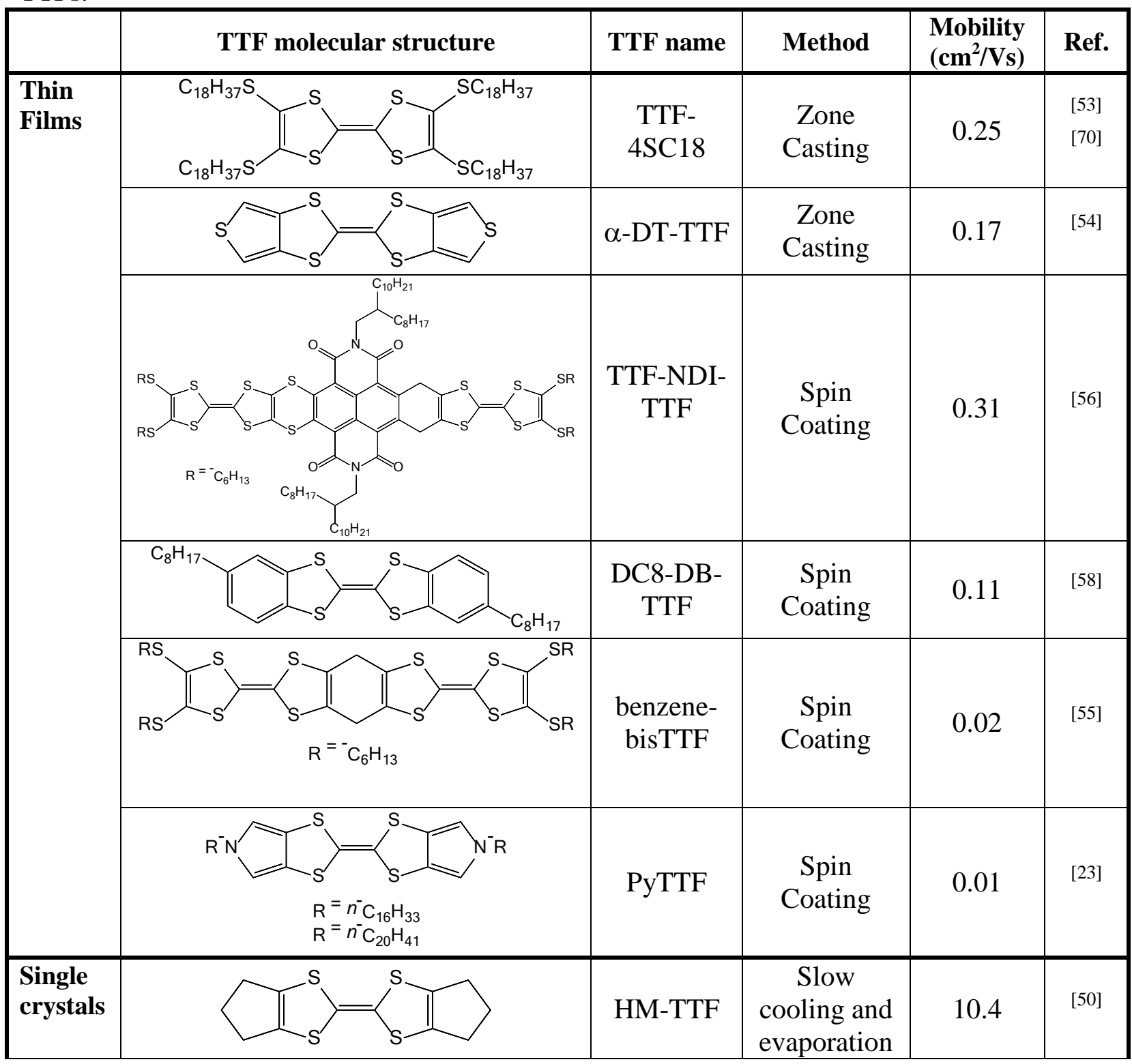




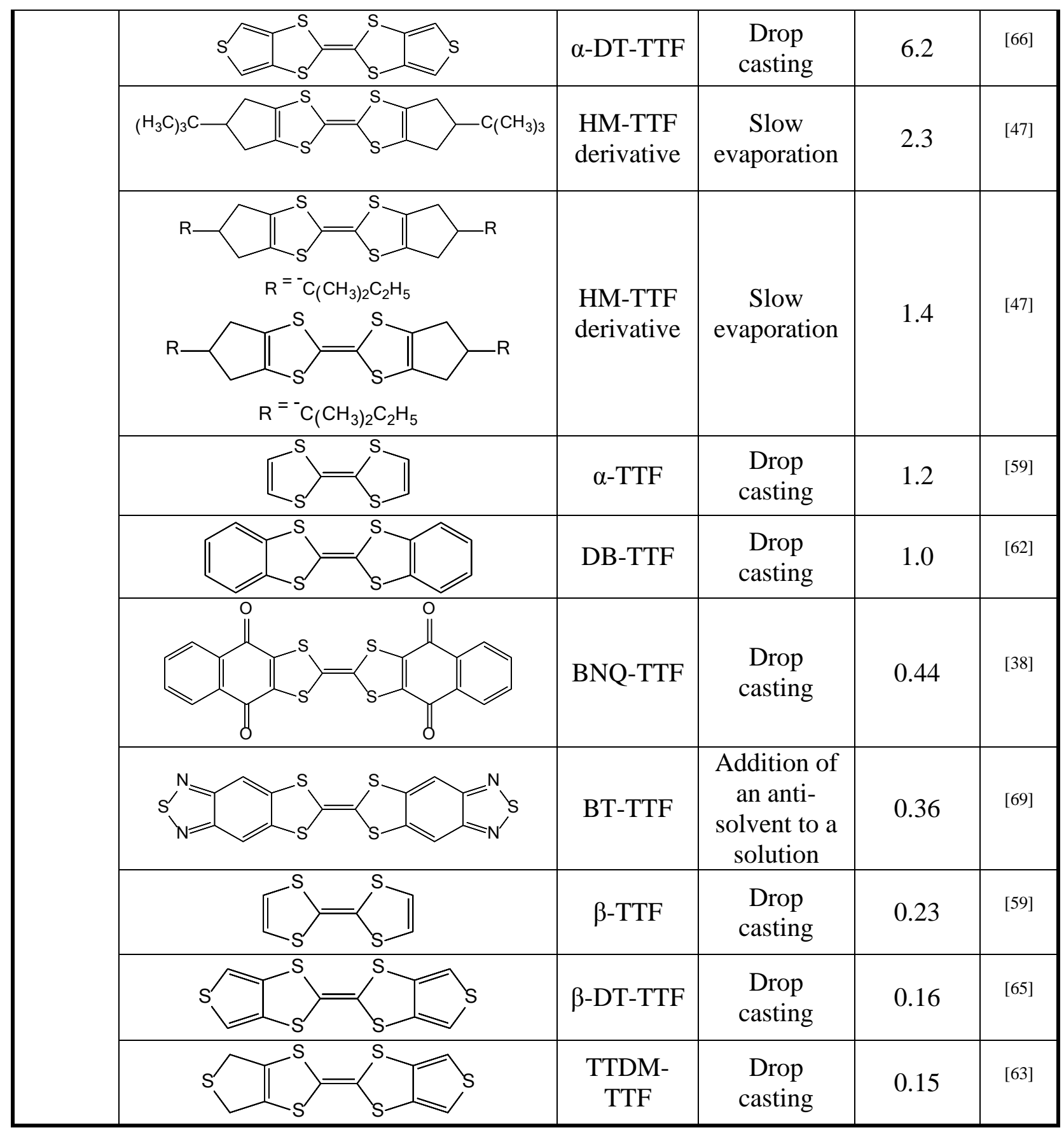

Representative electrical output and transfer characteristics are shown in Figure $\mathbf{3}$ for a thermally evaporated thin film of DB-TTF bisimide with top Au source and drain contacts. These devices exhibited mobilities in the range $0.12-0.40 \mathrm{~cm}^{2} / \mathrm{Vs}$ and on-off ratios of $10^{6}-10^{8}$. 

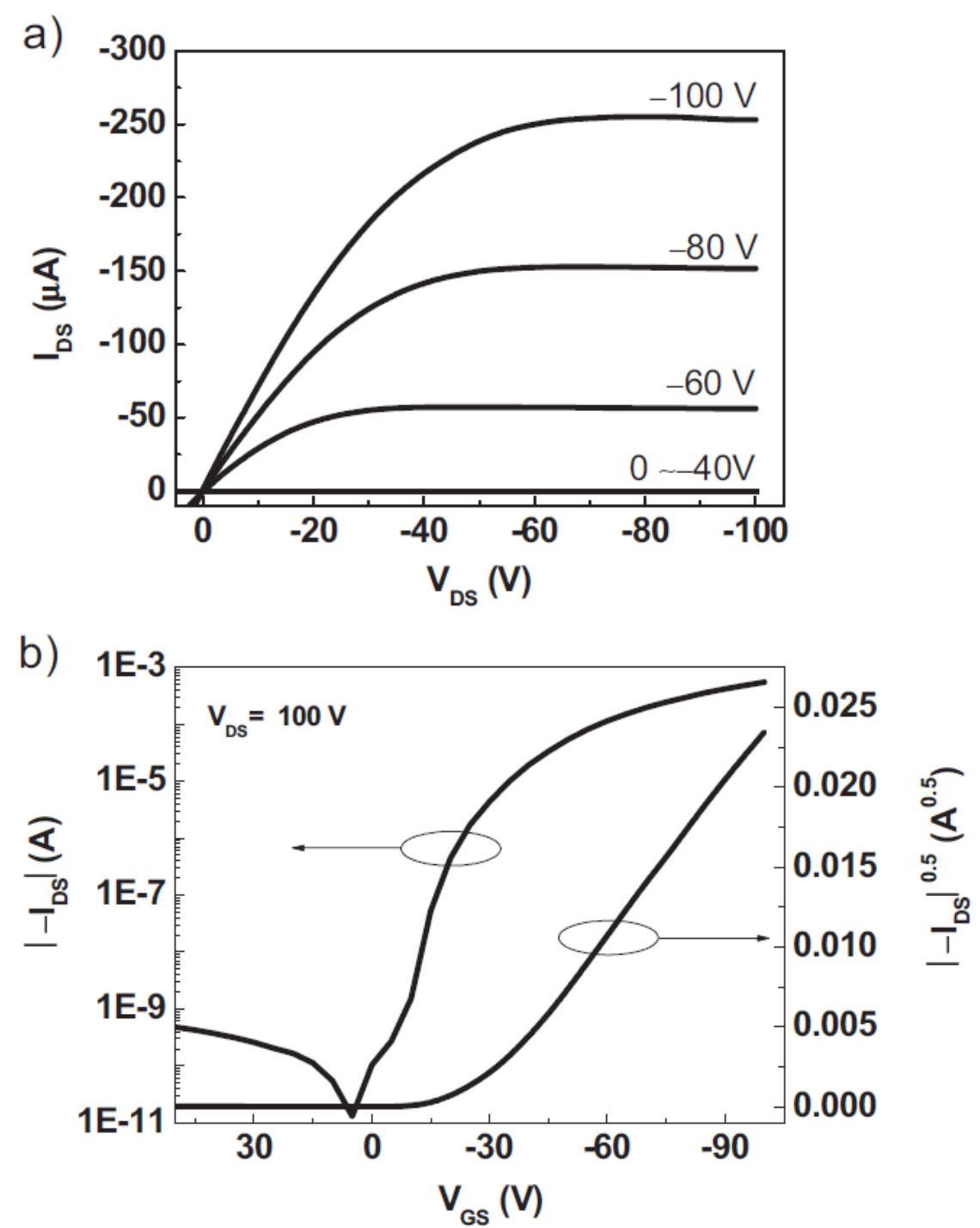

Figure 3. a) Output and b) transfer characteristics reported for an OFET based on DB-TTF bisimide evaporated thin film in a top contact configuration. Reproduced with permission from reference. ${ }^{[4]}$

Considering all the above, TTF derivatives have proven to be an important class of organic semiconductors suitable for most of the conventional deposition techniques exhibiting performance values comparable with other state-of-the-art materials. Field-effect mobilities, however, depend strongly on several parameters which have to be optimized to achieve high device performance. Related to this, devices based on TTF-derivatives have displayed a wide range of performances, reflected in mobility values that are scattered over several orders of 
magnitude. ${ }^{[21]}$ The following sections aim to provide more insights regarding some of the aspects that influence TTF device performance.

\section{Tuning crystal structure - Polymorphism}

The use of correlations between crystal structure and device performance as a means to understand transport mechanisms, and to facilitate the design of new promising organic semiconductors, is a complex subject. TTFs are a good platform to perform such correlation studies since it is possible to synthesize derivatives with very small variations in their molecular structure but which can significantly affect their solid-state organization.

An illustrative example of such an approach is the work by Saito and col. ${ }^{[71]}$ where they investigated the OFET properties of a family of TTF derivatives bearing four alkylthio groups with different chain lengths, namely TTSCn-TTF with $n=8,14$, and 18 . These systems were previously reported to exhibit a high conductivity for $n \geq 4$ due to the strong interchain interaction between the alkylchains that result in a fastener effect of the TTF moieties. ${ }^{\left[{ }^{[2]}\right.}$ Thin film OFETs were prepared by drop casting and it was seen that the mobility increased with the length of alkylchains up to $\sim 10^{-5} \mathrm{~cm}^{2} / \mathrm{Vs}$. This observation was in accordance with powder X-ray measurements that indicated that the distance between adjacent TTF moieties was lower when n was higher. Later on, OFET devices were also prepared with TTCn-TTF with $\mathrm{n}=12,18$, and 22 by zone casting and the same tendency was found. However, in this case, mobilities of up to $\sim 0.1 \mathrm{~cm}^{2} /$ Vs were achieved. ${ }^{[70]}$ This difference is probably due to the fact that the zone casting technique produces higher crystallinity films than drop casting. Further, since by zone casting the films prepared are anisotropic, it was observed that the mobility measured along the casting direction, which corresponds to the TTF stacking direction, was two orders of magnitude higher than when the measurements were performed in the perpendicular direction. ${ }^{[53]}$ 
Similarly, a set of bis-( $N$-alkylpyrrolo[3,4- $d]$ tetrathiafulvalene (PyTTF) derivatives with long alky chains were investigated as semiconductors when spin-coated on OFET structures. ${ }^{[23]}$ Again, shorter chains (i.e., n-butyl or octyl groups) gave a lower OFET mobility of $\sim 10^{-5}$ $\mathrm{cm}^{2} / \mathrm{Vs}$, while for derivatives with n-dodecyl or longer alkyl chains higher mobility values of up to $\sim 10^{-2} \mathrm{~cm}^{2} / \mathrm{Vs}$ were reached. By X-ray analysis, it was clear that in the former compounds the molecular overlap along the stacking direction is very small owing to the slipping of molecules along the long axis direction, although intermolecular $\mathrm{S} \cdots \mathrm{S}$ side-byside interactions are present. On the other hand, the molecules with long alkyl chains crystallize in a 2-D arrangement showing close stacking between PyTTF moieties as well as transverse intermolecular interactions through $\mathrm{S} \cdots \mathrm{S}$ contacts. The existence of a 2dimensional electronic layer is known to be a key requirement to achieve high OFET performance.

In addition to the investigation of the effect of the chain length of the TTF substituents, the influence of fusing heterocycles on the TTF core on the crystal packing has also been explored. It is widely known in the field of TTFs that the $\pi-\pi, S \cdots S$ and C-H $\cdots S$ interactions largely determine the solid-state arrangement of these materials. Thus, we previously reported a series of symmetric and asymmetric TTF molecules with fused five-member rings containing a sulphur atom. In the studied molecules the position of this heteroatom as well as the aromaticity of the ring was varied (Figure 4a). ${ }^{[63]}$ OFETs based on solution-grown single crystals of these molecules were then fabricated. It was observed that the derivatives with at least one aromatic fused ring with the sulphur atom in position 3 of the thiophene ring exhibited OFET mobilities of around 1 or 2 orders of magnitude higher. The single crystal Xray analysis revealed that molecules exhibiting higher performance crystallised in a herringbone pattern with short intra-stack as well as inter-stack molecular overlap (Figure 4a). In contrast, the rest of the derivatives crystallised following a brickwork-type motif, forming chains of quasi planar molecules interacting side-by-side that stack into layers. Transfer 
integral calculations also confirmed a higher intermolecular overlap in the herringbone-type crystals, in agreement with the OFET measurements. Noticeably, it was also found that asymmetric molecules showed poorer performance compared to the symmetric ones, and that the positional disorder caused by ethylenethio groups was detrimental to the device characteristics (i.e., leading to higher threshold voltages). Therefore, this study shows that, for this group of molecules, two types of 2-D electronic structures were formed and that the herringbone pattern is more favourable for electronic transport. Interestingly, $(\alpha)$ DT-TTF, which bears two aromatic rings but with the sulphur in position 2 of the thiophene ring, and a tert-butyl functionalised $(\alpha)$ DT-TTF were also investigated in transistors. However, these molecules crystallise in structures where the TTF moieties form trios or dimers, respectively, resulting in structures with a lower electronic dimensionality (Figure 4b-c). ${ }^{[37,40]}$ Accordingly, the OFET mobility of their thin films are much lower. 
a)

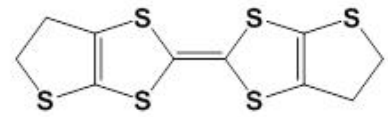

BET-TTF

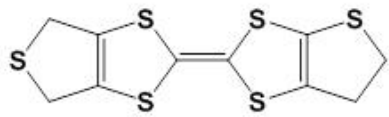

ETTDM-TTF
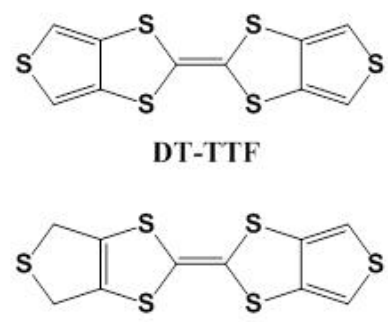

TTDM-TTF

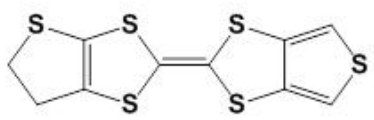

ETT-TTF

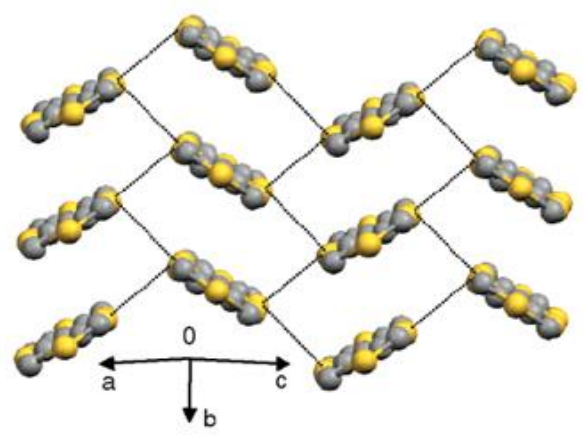

c)

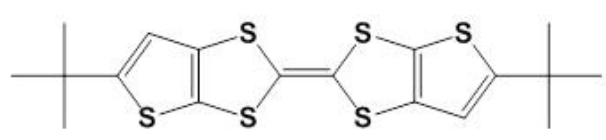

('Butyl)-( $\alpha)$ DT-TTF

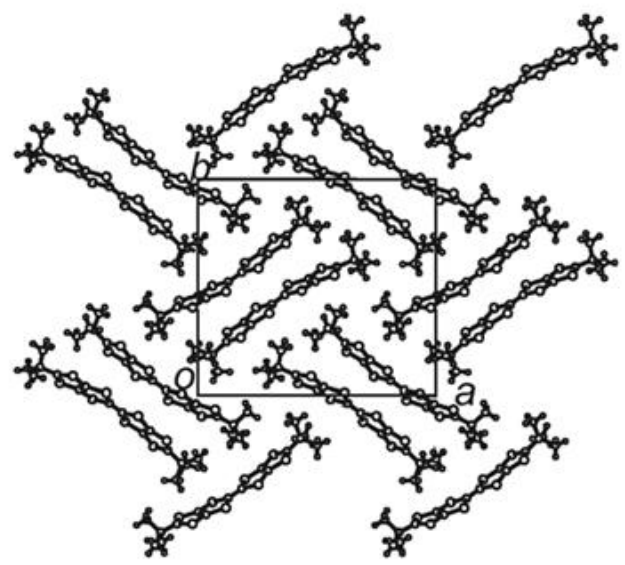

Figure 4. Molecular structures of the different TTF derivatives with fused S-heterocycles and packing motives. a) bis(ethylenethio)-tetrathiafulvalene (ethylenethio)(thiodimethylene)-tetrathiafulvalene (ETTDM-TTF),

(BET-TTF), tetrathiafulvalene (DT-TTF), thiophene)(thiodimethylene)-tetrathiafulvalene (TTDM-TTF), (ethylenethio)-(thiophene)-tetrathiafulvalene (ETT-TTF).

b) ( $\alpha$ )-dithiophenetetrathiafulvalene c) tert-butyl substituted ( $\alpha$ )-dithiophene-TTF. Reproduced with permission of references. ${ }^{[37,40,63]}$ 
Mori et al. also performed a structure-OFET performance correlation study of TTF derivatives functionalized with phenyl groups and found that variations in their herringbone structure affect the OFET mobility (Figure 5a). ${ }^{[41]}$ The highest mobility in evaporated thin-films was achieved for DB-TTF and DBP-TTF. The mobility of alkylphenyl TTFs dropped by about two orders of magnitude going from compound 3 to 5 of Figure 5 . In compound $\mathbf{7}$ it went a further two orders of magnitude down, and in $\mathbf{6}$ no OFET behaviour was measured. Single crystal X-ray revealed that while DB-TTF crystallises in an ideal two-dimensional network (Figure 5c), compounds $\mathbf{3}$ and $\mathbf{4}$ show only one-dimensional interactions due to the fact that the adjacent molecules in one direction of the herringbone plane are slipped along the long molecular axis (Figure 5d). This is probably caused by the bent structure of the molecules. In the case of compound $\mathbf{6}$, although the packing looks also like a herringbone structure, all neighbouring molecules are also slipped along the molecular axis, hence destroying the two dimensional contacts and leaving each TTF molecule highly isolated (Figure 5e). Finally, compound 7 was found to crystallise in zig-zag chains but only short $S \cdots S$ contacts were found within the chains, and not between chains (Figure 5b). This one-dimensional character could account for the lower OFET performance of this material. Despite all these studies, it should be kept in mind that the X-ray diffraction patterns of the thin films revealed that the crystal structure of the films were in some cases differing from the single crystal structure, or that in some films, there was more than one coexisting phase. In other correlation studies, similar findings related to the formation of crystal structures in thin films differing from the ones found in the resolved single crystal structures were reported. ${ }^{[73,74]}$ This highlights the complexity of controlling polymorphism, and the crucial importance of identifying the actual crystal structure of the processed semiconductor in the device to fully understand the relationship between structure and device performance. 


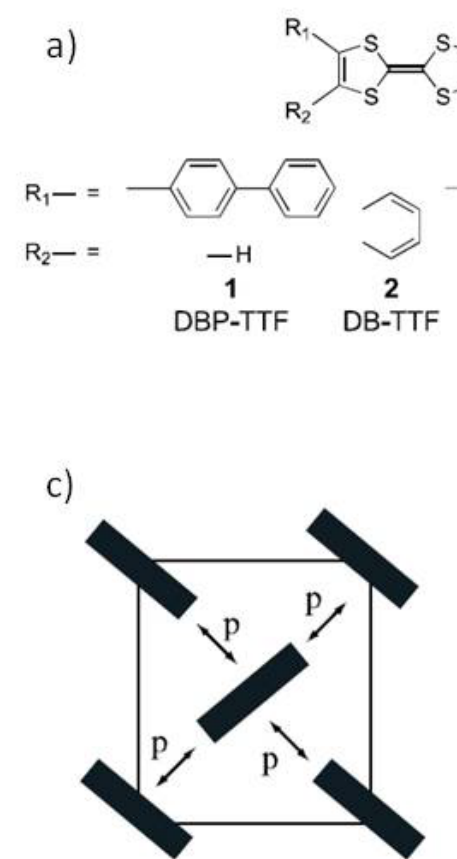

$$
I_{R_{1}}^{R_{2}}
$$

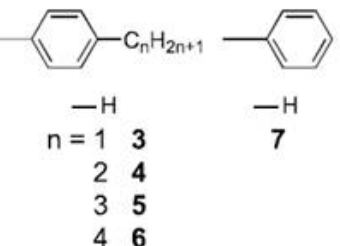

46

d)

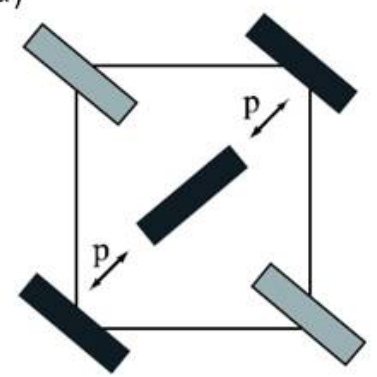

b)

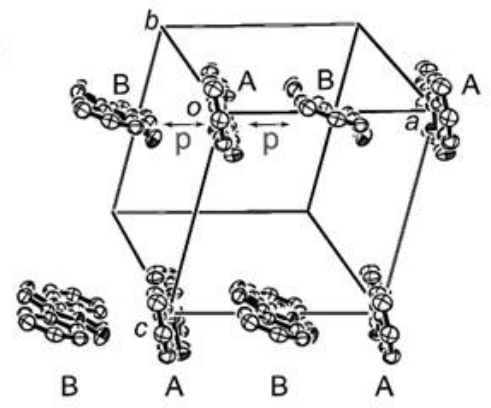

e)

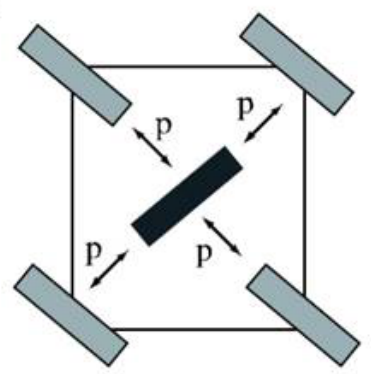

Figure 5. a) TTF derivatives functionalized with phenyl groups. b) Crystal structure of compound 7. Schematic representation of herringbone patterns found for compound 2 (DBTTF) (c), 3 and $\mathbf{4}$ (d) and $\mathbf{6}$ (e). The light grey molecules are slipped by a half molecular unit with respect to the dark molecules perpendicular to the sheet. Reproduced with permission from reference. ${ }^{[41]}$

Polymorphism is the ability of a given material to arrange atoms or molecules in more than one solid form or crystal structure. It is well known that, owing to the weak interaction energies, organic molecules are prone to polymorphic formation in the solid state and, therefore, polymorphism is a well-known phenomenon in organic materials. ${ }^{[75]}$ Organic semiconductors can therefore crystallise in a variety of polymorphs that can yield different device performance. The crystallographic description of polymorphs of benchmark organic semiconductors (i.e. oligothiophenes ${ }^{[75-84]}$ and acenes ${ }^{[84-95]}$ ) that can be formed varying the crystallisation methods have been published. However, works on the OFET performance of the different polymorphic modifications of a certain semiconductor are still very scarce. ${ }^{[60,84,96-101]}$ 
In the family of TTFs, two polymorphic forms for the parent TTF compound were reported more than twenty years ago. ${ }^{[102,103]}$ Recently, and taking into account the importance of the solvent in solution crystallization processes, OFETs based on these two phases have been fabricated crystallizing the material on octadecyltrichlorosilane (OTS) modified $\mathrm{SiO}_{2}$ substrates. ${ }^{[59]}$ Crystals of the pure $\alpha$-phase were obtained from $n$-heptane or $n$-hexane, whereas from chlorobenzene solutions the pure $\beta$-phase was produced. The maximum mobility achieved for the $\alpha$-phase was near $1.20 \mathrm{~cm}^{2} / \mathrm{Vs}$, while $\beta$-phase crystals exhibited a mobility of about one order of magnitude lower, reaching a maximum value of $0.23 \mathrm{~cm}^{2} / \mathrm{Vs}$ (Figure 6). The crystal structure of $\beta$-phase can be described as zig-zag chains sustained by S $\cdots S$ interactions. In contrast, the $\alpha$-phase has a herringbone motif with a short $b$-axis leading to a strong $\pi$-stacking along this direction and with short $S \cdots S$ inter-stack contacts, that is, shows a structure with higher bi-dimensionality, which is in accordance with the better performance of this polymorph (Figure 6).

a)

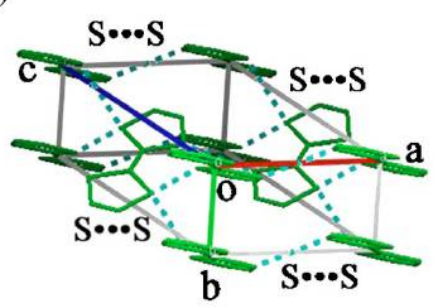

c)

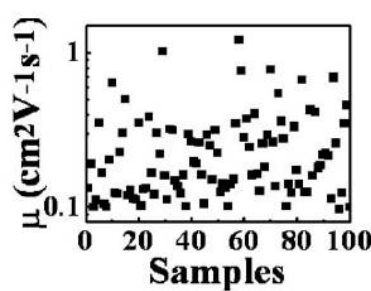

b)

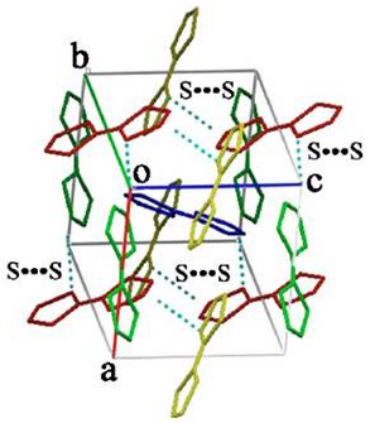

d)

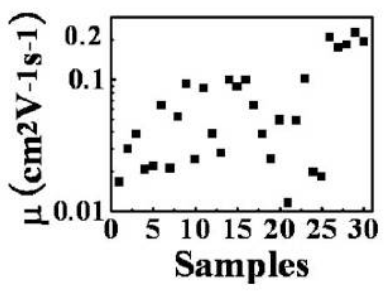

Figure 6. Crystal structure of the two polymorphic TTF phases $\alpha$ (a) and $\beta$ (b), and OFET mobility values obtained for $\alpha$ (c) and $\beta$ (d) polymorphs. Reproduced with permission from reference. $^{[59]}$ 
Further, dithiophene-tetrathiafulvalene (DT-TTF) has been acknowledged as one of the best performing TTF organic semiconductors, giving a mobility of up to $6.2 \mathrm{~cm}^{2} / \mathrm{Vs}$ in solution grown single crystals. ${ }^{[61,64,66,104]}$ However, it was observed that during the crystallization process, crystals with two morphologies coexist: some long plate crystals and some hexagonal ones. ${ }^{[65]}$ Lattice phonon confocal Raman spectroscopy and X-ray diffraction measurements confirmed that the different shaped crystals did indeed belong to two polymorphic forms, namely $\alpha$ - and $\beta$-DT-TTF. The former corresponded to the long plates and the phase that had been previously investigated in single crystal devices. These crystals crystallize in the monoclinic system, space group P21/a, with two centrosymmetric molecules per unit cell. ${ }^{[105]}$ The molecules arrange in a herringbone structure with the long axis tilted almost $20^{\circ}$ to $c$ axis and faced along $b$, the shortest crystal axis, which corresponds to the stacking direction of the molecules and, thus, where the $\pi-\pi$ interactions are maximized (Figure 4a). Although the single crystal structure of $\beta$-DT-TTF was not resolved, it was observed that this phase coincides with the one formed in evaporated thin films. The diffraction patterns of these films exhibited only the presence of peaks related to a periodicity of $13.18 \AA$, which is slightly larger than the (001) spacing of the $\alpha$-phase (13.11 $\AA$ ). By 2D-grazing incidence diffraction analysis and in out-of-plane geometries, it was concluded that this $\beta$-phase belongs to the same space group as the $\alpha$-phase and also follows a herringbone pattern. The device performance of these two modifications of DT-TTF crystals were studied in detail using solution prepared single crystal OFETs on $\mathrm{SiO}_{2}$ and Parylene $\mathrm{C}$ as dielectric. Mobility values between 0.6 and $1.2 \mathrm{~cm}^{2} / \mathrm{Vs}$, and between 0.03 and $0.17 \mathrm{~cm}^{2} / \mathrm{Vs}$, for the $\alpha$-DT-TTF and $\beta$-DTTTF, respectively, were achieved. This result is in agreement with the crystallographic studies that showed that, despite having two polymorphs with very close crystal structures, the $\alpha$ polymorph showed closer molecular packing along the crystalline $b$-axis. ${ }^{\text {[65] }}$ 
The complexity of polymorphism becomes clearly illustrated with the most studied TTF derivative in OFETs, DB-TTF. Single crystals OFETs reaching $1 \mathrm{~cm}^{2} / \mathrm{Vs}{ }^{[61,62]}$ have been reported for solution-processed DB-TTF single crystals, whereas in devices based on vacuum sublimed thin films, the field-effect mobility values reported range from $10^{-2} \mathrm{~cm}^{2} / \mathrm{Vs}$ to $10^{-1}$ $\mathrm{cm}^{2} / \mathrm{Vs}{ }^{[33,35,40,41]}$ Remarkably, up to four different polymorphs have been identified for this material, ${ }^{[106,107]}$ although only two of them have been investigated in OFETs: the thermodynamically more stable $\alpha$-phase, which was studied as single crystal, and the kinetically more favourable $\gamma$-phase, typically found in thin films

Considering all above, it has been shown with this family of semiconductors that minor modifications in the molecular structure might strongly affect the intermolecular interactions and, hence, the device performance. Additionally, it should be highlighted that some works have also shown that intramolecular interactions can also have a key role in controlling efficient molecular packing arrangement. ${ }^{[108,109]}$ That is, intramolecular interactions such as hydrogen bonding or intramolecular charge transfer might affect the molecule geometry which, concomitantly, induces changes in the solid-state organization. This approach has hardly been employed in this field, but could provide new tools for controlling the selfassembly of semiconductors. 


\section{Electronic modulation}

Carrying out synthetic modifications to an organic semiconductor core is not only a suitable route to tune the crystal packing but also constitutes a way to adjust the molecular electronic structure. As mentioned, TTFs molecules are intrinsically strong electron donors and, thus, they are potential p-channel organic semiconductors. However, very often TTFs show HOMO energy levels above $-4.9 \mathrm{eV}$, which results in a poor stability of these materials in ambient conditions. Many thin films of TTFs become doped by oxygen exposure, which is then reflected in the OFET characteristics with high off currents (i.e., low on/off ratio) and positive threshold voltages. For instance, DB-TTF gives rise to devices with good OFET mobility but that are extremely unstable in air. To overcome this, a few research groups have focused on synthesising DB-TTF derivatives with electron-withdrawing groups in order to shift down the HOMO levels. In this direction, electron deficient heterocycles such as imides ${ }^{[44,110,111]}$ and 2,1,3-chalcongendiazole ${ }^{[73,74]}$ rings have been fused to the DB-TTF core (Figure 7a). The HOMO levels were lowered to values in the range from -5.1 to $-5.3 \mathrm{eV}$, giving rise to devices that exhibited a high performance and, importantly, good ambient stability during several weeks.

Attaching electron acceptor groups to TTFs in addition to diminish the HOMO level to some extent, also results in a more important decrease of the LUMO. It is generally assumed that the LUMO level has to be lower than $-3.5 \mathrm{eV}$, or preferably $-4.0 \mathrm{eV}$, to ensure electron injection and stability at ambient conditions. ${ }^{[110]}$ Further, for hole injection, typically the HOMO level has to be between -4.9 and $-5.5 \mathrm{eV} \cdot{ }^{[112]}$ Hence, with balanced orbital energy levels and efficient HOMO-HOMO and LUMO-LUMO overlap, donor-acceptor systems are potential candidates as ambipolar semiconductors. The first example of an ambipolar TTF material was found for bis(naphthoquinone)-TTF (BNQ-TTF) (Figure 7b). ${ }^{[38]}$ This derivative had an HOMO and LUMO level at $-5.20 \mathrm{eV}$ and $-3.43 \mathrm{eV}$, respectively. The OFET mobility 
measured in nitrogen atmosphere was around $10^{-2} \mathrm{~cm}^{2} / \mathrm{Vs}$ for holes and $10^{-4} \mathrm{~cm}^{2} / \mathrm{Vs}$ for electrons, but in air the devices only showed p-channel transport. Similar results showing ambipolar transport were obtained for the dyad formed by a 4,8-dicyano substituted benzothiazole fused to a TTF (CN-BTD-TTF): $\left.\mu_{\mathrm{h}} \sim \mu_{\mathrm{e}} \sim 10^{-5} \mathrm{~cm}^{2} / \mathrm{Vs}\right) .{ }^{[113]}$ Further, largely extended $\pi$-conjugated electron deficient moieties that have been proved to behave as efficient n-channel semiconductors such as perylenediimide (PDI) and naphtalenediimide (NDI), have also been fused to TTFs (Figure 7b). ${ }^{[56,114]}$ In both resulting dyads the HOMOLUMO gap was significantly reduced to $1.1 \mathrm{eV}$ for PDI-TTF and $0.8 \mathrm{eV}$ for NDI-TTF derivatives. Thin films of these donor-acceptor systems prepared from solution-coating showed ambipolar behaviour reaching mobilities in air of $\mu_{\mathrm{h}}=0.03 \mathrm{~cm}^{2} / \mathrm{Vs}$ and $\mu_{\mathrm{e}}=0.003$ $\mathrm{cm}^{2} / \mathrm{Vs}{ }^{[56]}$

Interestingly, the introduction of halogen groups or trifluoromethyl groups to quinoxalinoTTF has resulted in turning a presumably p-channel material into an n-channel material (Figure 7c). ${ }^{[28,115]}$ High electron mobilities of the order $0.01-0.11 \mathrm{~cm}^{2} /$ Vs were realised with these materials. The loss of the p-type characteristics can be rationalised by the large shifting of the HOMO energy levels below $-5.5 \mathrm{eV}$, preventing efficient hole injection. By modifying the number of halogen atoms or by replacing the trifluoromethyl group with a methyl group, the frontier energy levels were shifted upwards and only p-channel transport was observed in the OFET devices. Thus, these results demonstrated that through chemical synthesis the OFET polarity can be determined. 


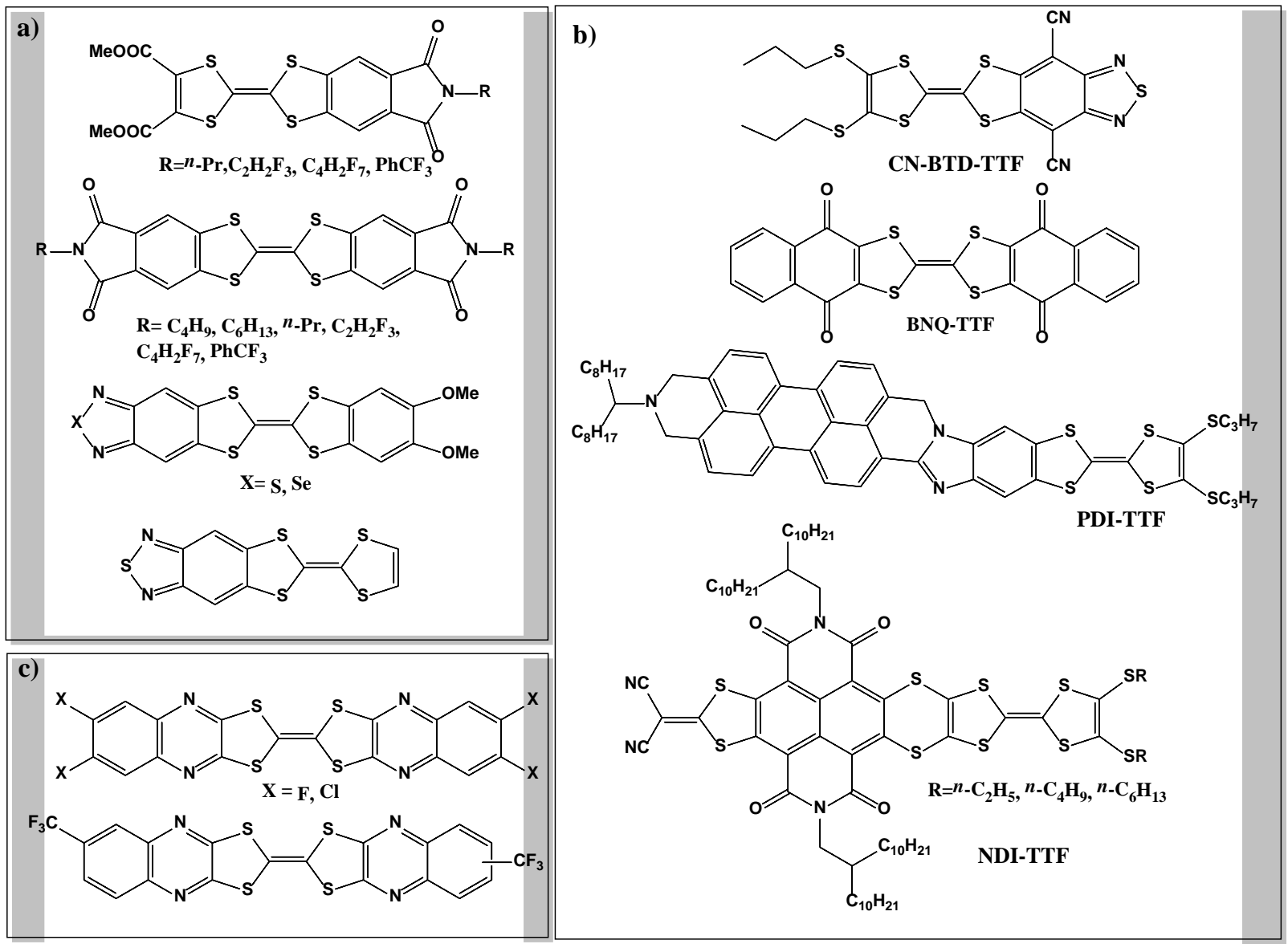

Figure 7. a) TTF derivatives with electron-deficient groups that promote ambient stability to the resulting devices. b) TTF derivatives that exhibit ambipolar OFET characteristics. c) TTF derivatives that behave as n-type semiconductors.

It has been argued that as long as considerable HOMO-HOMO and LUMO-LUMO electronic interactions are present, hole and electron conduction are both generic properties of organic semiconductors. However, the observation of such ambipolar behaviour in OFETs depends on the matching of the energy levels between the HOMO and LUMO of the molecule and the metal workfunction for ensuring charge injection. ${ }^{[116,117]}$ DT-TTF shows a low lying HOMO level of about $-4.9 \mathrm{eV}$, and a high lying LUMO of about $-1.2 \mathrm{eV} .^{[118]}$ The large bandgap, mostly related to the high LUMO energy level in this material, is a limitation for efficient electron injection and is related to n-channel behaviour. Theoretical calculations, however, demonstrate that efficient and balanced HOMO-HOMO and LUMO-LUMO interactions can 
be found in $\alpha$-DT-TTF crystal. ${ }^{[119]}$ Kelvin Probe Microscopy (KPM) experiments carried out in single crystal $\alpha$-DT-TTF OFET devices during operation in air have shown evidence of intrinsic ambipolar transport in this material. ${ }^{[119]}$ Figures 8a shows the effective potential drop following the channel recorded as a function of the gate bias (Figure 8b shows the associated profiles). The observation of S-shaped potential curves of the transistor channel is indicative of ambipolar transport, although OFETs devices only exhibited p-channel behaviour. This finding supports the idea that n-transport is not limited to materials with high electron affinity and that macroscopic electrical aspects such as contact resistance might mask the observation of the material intrinsic transport properties.

(a)

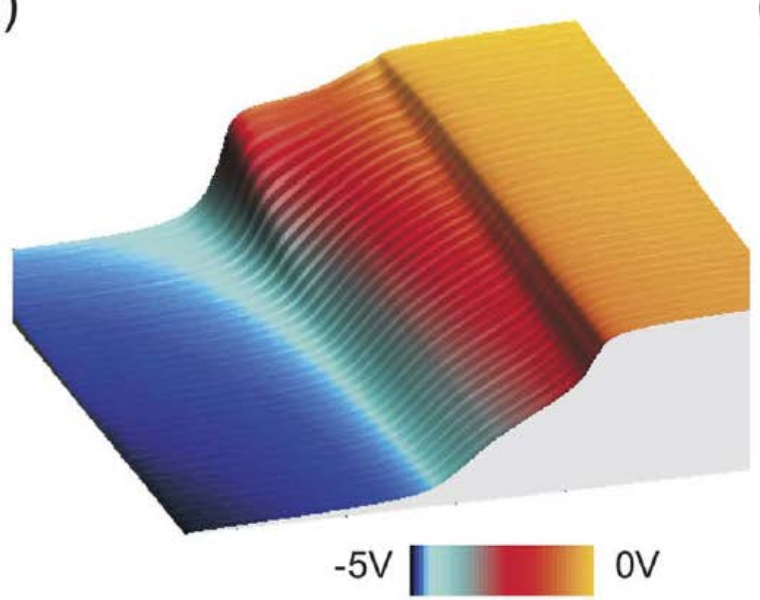

(b)

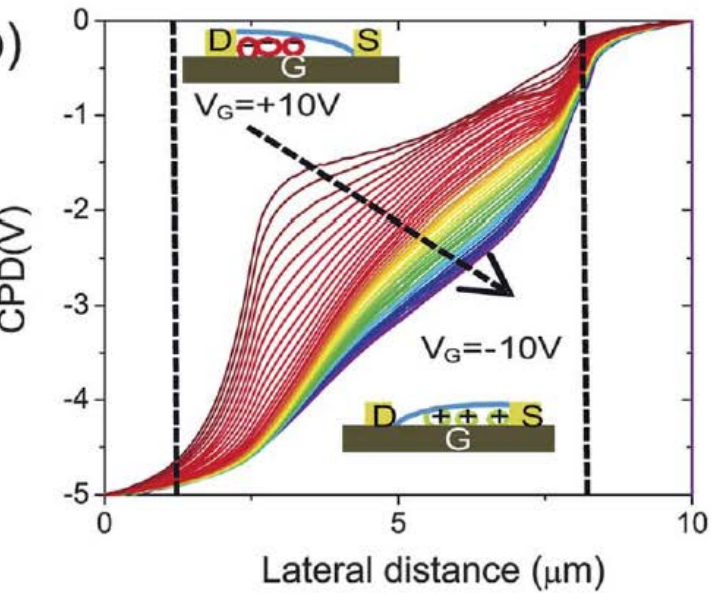

Figure 8. Ambipolar behaviour of a DT-TTF single crystal OFET in the transistor channel. (a) Three-dimensional view of the contact potential difference (CPD) along the transistor channel for gate voltages from $\mathrm{V}_{\mathrm{G}}=+10 \mathrm{~V}$ to $-10 \mathrm{~V}$ in steps of $0.5 \mathrm{~V}$ and a constant drain voltage of $V_{D}=-5 V$. (b) CPD profiles with the edge of source and drain indicated by dashed lines. The inset gives a schematic distribution of the charge carriers in the transistor channel. Reproduced with permission from reference. ${ }^{[119]}$ 


\section{Theoretical modelling of charge transport in TTF derivatives}

Theoretical efforts to understand the relatively high room temperature mobilities observed in TTF-based OFETs based have primarily focussed on thermally induced hopping of localised charge carriers. For organic molecular crystals, this typically entails using electronic structure methods (e.g. Density Functional Theory) to calculate the relevant terms according to the semi-classical Marcus theory ${ }^{[120]}$ which describes this type of transport mechanism. Specifically, the charge transfer rate $(k)$ between adjacent TTF molecules is predicted to be given by:

$k=\frac{J^{2}}{h}\left(\frac{\pi}{\lambda k_{B} T}\right)^{1 / 2} \exp \left(-\frac{\lambda}{4 k_{B} T}\right)$,

where $J$ is the electronic coupling transfer integral between the charge donating molecule and the charge accepting molecule, $\lambda$ is the reorganisation energy of the system associated with charge transfer, and $k_{\mathrm{B}}$ and $T$ are the Boltzmann constant and the temperature, respectively.

Clearly, in this model, $J$ should be increased and $\lambda$ should be decreased for maximising charge mobilities. Both $J$ and $\lambda$ can, and usually do, differ depending on whether the transport involves hole-like or electron-like carriers. Although $\lambda$ is a characteristic single value for any particular crystal, $J$ is a variable which generally depends on the relative positions and orientations of neighbouring molecules and the shapes of their frontier. ${ }^{[121]}$ As such, the

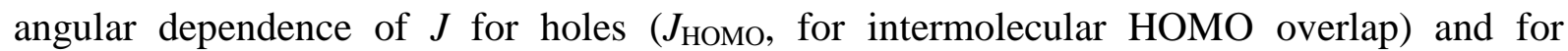

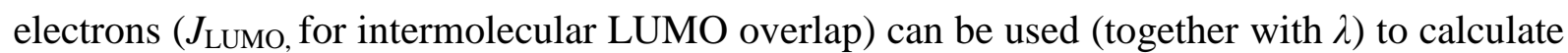
the respective favoured directions for charge transport through the respective crystal. Such plots have been used for TTF-derivative crystals for helping to rationalise experimental mobility data, ${ }^{[38,111,122]}$ and to predict the extent of anisotropy of hole and electron mobilities in as-yet synthesized TTF-derivative compounds. ${ }^{[123,124]}$ The wide range of calculated behaviours stems from the many synthetic options open for chemical, and thus electronic 
modification of TTF-derivative molecules and the large number of crystal packings that they can potentially exhibit. As mentioned above, of particular interest in this context is the recent discovery of TTF-derivative compounds which may have suitable transport properties of for use as ambipolar semiconductors (i.e. able to efficiently and stably transport both holes and electrons). ${ }^{[38,111]}$ In Figure 9 we show an example of theoretically derived directional charge mobility plot for DB-TTF bisimide; a potential ambipolar TTF-derivative.
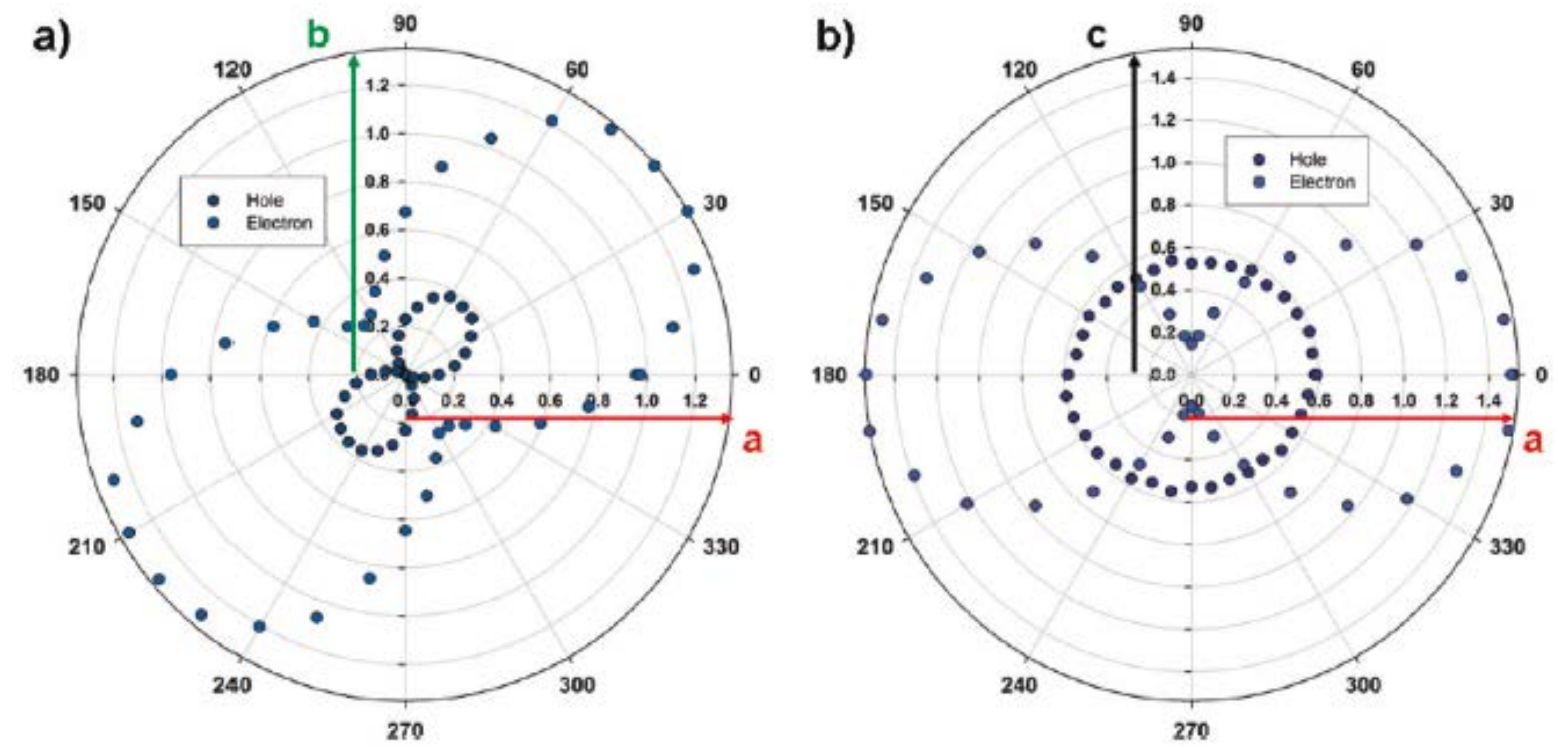

Figure 9. Anisotropy of hole and electron mobilities of DB-TTF bisimide calculated in: a) the a-b plane, and b) the a-c plane. Taken with permission from reference. ${ }^{[111]}$

At $0 \mathrm{~K}$ the intermolecular interactions described by $J$ would be undisturbed throughout the crystal allowing for full delocalisation of charge carriers, thus entailing band-like transport. At finite temperatures, thermal disorder causes the intermolecular couplings to fluctuate. This modulates the values of $J$ between all molecules in all directions, tending to localise the charge carriers. ${ }^{[125]}$ Application of the Marcus theory to organic molecular crystals has tended to assume full localisation of the charge carriers on individual molecules. Clearly, however, for compounds such as TTF-derivatives, the electronic coupling helping to drive the observed high mobilities should also be high, thus promoting locally delcocalised charge carriers which 
should be more resilient to thermal disruption. Currently, there is no direct experimental evidence to assess the degree of charge carrier delocalisation in TTF-derivatives. Measurements on pentacene, an organic molecular compound with comparable mobilities to the best performing TTF-derivatives, however, have confirmed that each carrier is delocalised over $\sim 10$ molecules. ${ }^{[126,127]}$

The degree of delocalisation of the charge carriers in TTF-derivatives, and, more generally, the role of the extended molecular environment on charge transport, is particularly important for calculations of $\lambda$. Typically, however, one estimates the full reorganisation energy associated with the charge transfer process, $\lambda_{\text {tot }}$, by calculating the internal fraction, $\lambda_{\text {int }}$ (i.e. the local molecular part) by either: (i) the energy change that occurs when a relaxed charged molecule interchanges a charge with a relaxed neutral molecule and both subsequently structurally relax, or (ii) the sum of the shifts in structural displacements due to characteristic normal modes going from the molecular charge donor to the molecular charge acceptor. Although TTF-derivative molecules tend to be planar when in a crystal, many TTFderivative molecules in free space adopt a boat like conformation when neutral, and are only planar when positively charged. ${ }^{[128]}$ The use of only a single unconstrained TTF-derivative molecule to calculate $\lambda_{\text {int }}$ will thus include this non-crystal-like structural relaxation leading to spuriously high $\lambda_{\text {int }}$ values. One suggested way to indirectly incorporate the effects of molecular packing, and thus to get a better estimate of $\lambda_{\text {int }}$ within a crystal, is to constraint the all atoms of neutral TTF molecules to move only in a single plane. ${ }^{[129]}$ This constraint has since been adopted by other works using both (i) and (ii) approaches. ${ }^{[123,130-132]}$ Calculation of $\lambda_{\text {int }}$ using approach (ii) can also be performed using only experimental molecular vibrational data provided from Raman spectra. Such an experiment has been done for the DT-TTF crystal giving a $\lambda_{\text {int }}$ value of $0.220 \mathrm{eV}$ which matches well the theoretical value from DFT calculations $(0.243 \mathrm{eV})$ using approach (i) and the molecular planarity constraint. ${ }^{[130]}$ 
Although structural constraints thus provide an experimentally verified means to provide reasonable theoretical estimates of $\lambda_{\text {int }}$ for individual TTF-derivative molecules in a crystal, the full reorganisation energy, $\lambda_{\text {tot }}$, includes contributions from long range structural polarisation (and subsequent electronic relaxation). The degree of structural polarisation is usually linked with the degree of charge delocalisation with more delocalised charge carriers tending to be less polarising and vice versa. Alternatively, we can say that between the extremes of fully delocalised band transport and highly localised molecular polarons, there is a range of charge carrier possibilities which can be thought of as partially delocalised polarons. To explore the effect of charge delocalisation, DFT calculations in which a single charged molecule is embedded in between two rigid neighbouring DT-TTF molecules which are fixed in their crystal positions have been performed. The resulting charge delocalisation away from the embedded molecule leading to a more neutral-like geometry and an $82 \%$ drop to $0.042 \mathrm{eV}$ of $\lambda_{\text {int }}$ (not $\lambda_{\text {tot }}$ as no environmental structural relaxation is permitted) with respect to that calculated for a planar single molecule. ${ }^{[129]}$ The resulting $\lambda_{\text {int }}$ is more in line with $\lambda_{\text {int }}$ values from other high mobility organic molecular crystals such as pentacene. ${ }^{[133]}$ tending to support the occurrence of this phenomenon. We note that similar reductions $\lambda_{\text {int }}$ in have been calculated for TTF and BDH-TTP using structurally fixed embedding molecules. ${ }^{[134]}$ The extent of charge delocalisation in such models can be varied via use of greater or less HartreeFock exchange in the calculations, tending to localise and delocalise charge on the central molecule respectively. Calculating $\lambda_{\text {int }}$ using same three molecule embedding set-up but with more localised charge on the embedded molecule also results in a large relative decrease in $\lambda_{\text {int}}$, but mainly due to electronic polarisation. ${ }^{[118]}$ Unlike delocalisation, however, the reduction in $\lambda_{\text {int }}$ due to electronic polarisation is found to be highly susceptible to disruption by thermal disorder and thus partial delocalised polaronic carriers are probably more likely to be the thermally activated carriers in TTF derivatives. Explicit modelling of the effects of partially delocalised carriers in an extended structurally and electronically responsive 
environment is computationally very demanding for electronic structure calculations and thus direct estimates of $\lambda_{\text {tot }}$ from theory are currently unavailable. Simple general models using a dielectric continuum have estimated the environmental contribution to $\lambda_{\text {tot }}$ to be $0.2-0.3 \mathrm{eV}$ for a typical organic molecular crystal. ${ }^{[135]}$ For DT-TTF a few studies have used models containing two or more structurally flexible embedding molecules which, in principle, are able to incorporate a proportion of $\lambda_{\text {tot. }}{ }^{[118,136,137]}$ Calculated parameters from such models tend to support the experimental mobility measurements. Such calculations better reflect the complex interactions in real TTF-derivative samples and will permit a better validation of theoretical methods in order to further improve our fundamental microscopic perspective into the nature of the charge carriers and their transport in TTF-derivatives. 


\section{Source-Drain contacts}

The field-effect mobility extracted in a transistor is not an intrinsic parameter of an organic semiconductor but a device parameter. Certainly, the same material, depending on how is integrated and the configuration of the device, can give rise to different OFET mobility values. Contact resistance can play an important role in device performance. This has been observed also in TTF OFETs. Thin film OFETs of DB-TTF were investigated using different source and drain metal materials: $\mathrm{Au}, \mathrm{Ag}, \mathrm{Cu}$ and the organic charge transfer (CT) salt (TTF)(TCNQ), where TCNQ stands for tetracyanoquinodimethane. ${ }^{[42]}$ The mobility and also the contact resistance were found to change with the metal workfunction. The organic metal resulted in the lower contact resistance devices, which was attributed to small potential shift on the organic/organic interface compared with the organic/metal interface. ${ }^{[138]}$ In agreement with these results, OFETs based on single crystals of hexamethylene-tetrathiafulvalene (HMTTF) exhibited a mobility of $0.02 \mathrm{~cm}^{2} /$ Vs when Au was employed as source-drain but reached a mobility exceeding $10 \mathrm{~cm}^{2} /$ Vs when gold was replaced by (TTF)(TCNQ). ${ }^{[50]}$

Single crystals of DT-TTF were also grown on evaporated (TTF)(TCNQ) electrodes by drop casting a solution of the material in either toluene or chlorobenzene. ${ }^{[66]}$ It was observed that the average OFET mobility in devices prepared from $\mathrm{PhCl}$ solutions reached a value of $2.5 \mathrm{~cm}^{2} / \mathrm{Vs}$ (maximum value of $6.2 \mathrm{~cm}^{2} / \mathrm{Vs}$ ), which was four-fold more than that obtained from devices prepared from solutions of DT-TTF in toluene. The latter devices showed mobilities only slightly higher than when gold was used as contact. These discrepancies in performance were attributed to differences in the contact resistances as demonstrated by KPM measurements. The utilization of a more polar solvent (i.e., PhCl) led to a certain re-dissolution of the organic metal with a subsequent co-precipitation of the two materials (i.e., organic metal and organic semiconductor). This result therefore highlights the 
importance of the contacts in OFETs, not only in terms of energy alignment, but also with respect to the interface morphology.

Considering that the conducting charge transfer salts are based on TTFs, Mori et al. introduced the concept of “self-contacting” TTF OFETs. This was achieved by evaporating TCNQ through a shadow-mask on thin films of tetramethyl-TTF or HM-TTF. ${ }^{[30,31]}$ The areas in which the TCNQ was evaporated on the TTF derivative were conducting due to the formation of a CT salt, whereas the remaining regions of the film were used for the active material. A similar approach was used to evaporate the organic semiconductor on prepatterned TCNQ films. All these devices exhibited a very low contact resistance and high mobility. This has also been successfully realized by ink jet printing a solution of TCNQ on a HM-TTF evaporated thin film. ${ }^{[139]}$ It was observed that the chosen solvent has to dissolve the dopant well (in this case, TCNQ) but only poorly dissolve the organic semiconductor in order to have an appropriate reaction rate for the formation of the charge transfer complex salt. If the reaction is too fast the resulting morphological connection between the electrode and the semiconductor is worse. The authors claim that this method guarantees a good energy matching since the Fermi level of the electrode will be located between the HOMO of the TTF derivative and the LUMO of TCNQ, and as a consequence, the Fermi level will be close to the HOMO level of the active material (Figure 10). 


\section{Electrode Active layer}

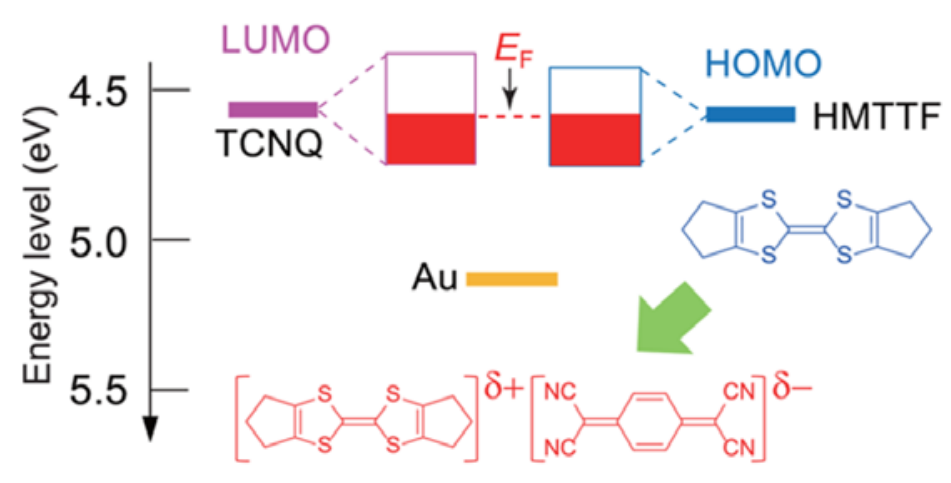

Figure 10. Energy level alignment in "self-contact" transistor. HM-TTF as active material and TCNQ as dopant to form a CT conducting salt that serves as source-drain electrodes. Reproduced with permission from reference. ${ }^{[139]}$

\section{Other perspectives}

An appealing field of applications for organic-based devices that has become very attractive in the last decades is sensors. ${ }^{[140]}$ Single crystal and thin film TTF OFETs were previously demonstrated to exhibit a large photoresponsivity, and therefore, light can operate as an additional gate to modulate the density of charge carriers. ${ }^{[141,142]}$ Such devices could be suitable for the detection of light. In this context, a $\pi$-conjugated donor-acceptor dyad composed of a tetrathiafulvalene-fused perylenediimide (TTF-PDI) was synthesised. Interestingly, wavelength-dependent photo-response measurements of the TTF-PDI dyad measured in an OFET configuration resembled its absorption profile extracted from a thinfilm coated on a transparent quartz substrate (Figure 11). ${ }^{[114]}$ A significant photo-response at an energy corresponding to PDI-localized electronic $\pi-\pi^{*}$ transitions was observed (energy range from 2.2 to $2.5 \mathrm{eV}$ ) and, further, a more moderate effect due to an intramolecular charge transfer from the HOMO localized on the TTF unit to the LUMO localized on the PDI moiety 
was also detected (between 1.1 and $1.5 \mathrm{eV}$ ). This work clearly elucidates the interplay between intra- and intermolecular electronic processes in organic devices and opens the door to the exploitation of such devices for wavelength selective photo-detectors.

Materials able to detect chemical vapours are also attractive for niche applications where organic materials can potentially outperform their classical counterparts. With TTF OFETs the detection of DECP (diethyl chlorophosphate) or $\mathrm{POCl}_{3}$, stimulants of phosphatebased nerve agents, have been demonstrated. In these devices the off-current was used as output of the sensor, and levels down to 10 parts per billion (ppb) of these vapours could be effectively detected. The same system was further used to detect low concentrations of TNT (100 ppb) since the saturation current and hole mobility decreased when the devices interacted with the TNT vapours. ${ }^{[46]}$ 
(a)
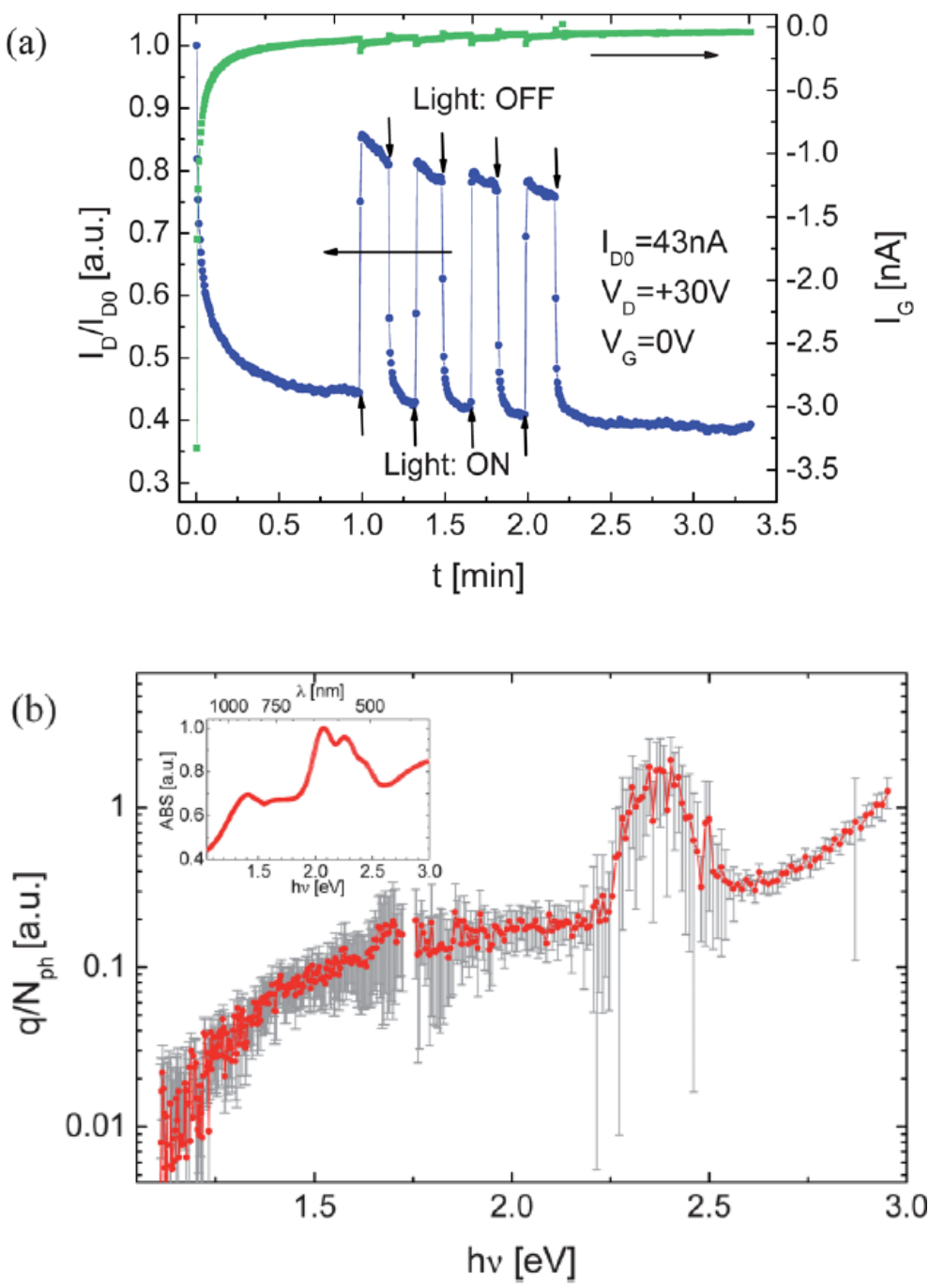

Figure 11. External photon-to-photogenerated charge conversion efficiency as a function of incident photon energy. a) OFET Photo response using light of a solar simulator at 100 $\mathrm{mW} / \mathrm{cm}^{2}$. b) Spectrum measured for a TTF-PDI thin-film OFET device and a solution processed thin-film on a quartz slide (inset). Reproduced with permission from reference. ${ }^{[114]}$

Due to the fact that they are good electron donors, TTFs can also be used as n-type doping in organic semiconductors. ${ }^{[143]}$ In agreement with the standard model commonly used for crystalline semiconductors, controlled doping can have a strong impact on the electrical characteristics. The device performance of the n-channel organic semiconductor, N,N'-di((Z)9-octadecene)-3,4,9,10-perylene tetracarboxylic diimide has been dramatically increased by a factor of up to 30 by blending it with TTFs. ${ }^{[144]}$ However, this effect was attributed more to a 
structural modification rather than an electronic one. Indeed, the off-currents in these devices where not significantly affected by the blends, indicating that the conductivity was not altered. Hence, the enhanced mobility was attributed to the facilitated charge transport due to the increased stack-ordering and crystallinity as well as decrease in grain boundaries in the spincoated films.

TTF derivatives have also been extensively used as the donor component of charge transfer salts for over forty years. Such conducting salts have also been more recently applied as metal contacts in organic transistors. Although this is beyond the scope of this review, we recommend references ${ }^{[145]}$ and ${ }^{[138]}$ to the reader, where the use of charge transfer salts in devices is reviewed.

\section{Conclusions}

TTF derivatives are an appealing class of organic small molecules and are among the most investigated organic semiconductors for organic electronics. They are fascinating due to their high performance, their easy processability, and the possibilities they offer to be chemically modified for specific applications. It has been shown in this work that the properties of TTFs can be adapted to the processing needs of different fabrication methods and high OFET mobility values have been achieved both in vacuum deposited and in solution processed materials. Due to fact that different derivatives can be relatively easy prepared, they can be used as model organic semiconductors systems in order to understand the influence of the crystal structure on the device performance. However, it should be taken into account that in thin films of organic semiconductors different polymorphs might coexist, and therefore, the comparison of single crystal OFETs can give us a better understanding of the structureproperty correlation. In this sense, TTFs also are a promising platform since they can generally be easily crystallised from solution. Furthermore, their electronic structure can be also tuned in order to provide ambient stability or even to modify they behaviour in devices 
resulting in p-channel, n-channel or even ambipolar transport characteristics. From a fundamental point of view, TTFs have been a subject of study for many years in organic electronics, but they might also show great potential in novel niche applications as for instance in sensor devices. Taking all into account, to move towards applications the challenge now is to find solution processed methodologies that permit to fabricate stable devices with an imperative control of the crystal phase formed and film morphology to achieve high reproducibility.

\section{Acknowledgements}

The authors thank the ERC StG 2012-306826 e-GAMES project, the Networking Research Center on Bioengineering, Biomaterials and Nanomedicine (CIBER-BBN), the DGI (Spain) with projects BE-WELL CTQ2013-40480-R and MAT2012-30924, and the Generalitat de Catalunya (2014-SGR-17, 2014SGR97 and XRQTC).

\section{References:}

[1] J. E. Lilienfeld, U.S. Patent 1,745,175 1930.

[2] W. B. Shockley, J. Bardeen, W. H. Brattain, Nobel Prize in Physics 1956.

[3] Z. I. Alferov, H. Kroemer, J. S. Kilby, Nobel Prize in Physics 2000.

[4] D. Kahng, M. M. Atalla, In Silicon-silicon dioxidefield induced surface devices; IRE Solid-State Devices Research Conference, Pittsburgh, PA, 1960.

[5] G. Horowitz, D. Fichou, X. Peng, Z. Xu, F. Garnier, Solid State Commun. 1989, 72, 381.

[6] H. Koezuka, A. Tsumura, T. Ando, Synth. Met. 1987, 18, 699.

[7] http://www.plasticlogic.com/.

[8] http://www.lg.com/us/mobile-phones/gflex.

[9] http://www.samsung.com/us/oled-tv/.

[10] A. Shehu, S. D. Quiroga, P. D’Angelo, C. Albonetti, F. Borgatti, M. Murgia, A. Scorzoni, P. Stoliar, F. Biscarini, Phys. Rev. Lett. 2010, 104, 246602.

[11] C. Wang, H. Dong, W. Hu, Y. Liu, D. Zhu, Chem. Rev. 2012, 112, 2208.

[12] R. D. McCullough, Adv. Mater. 1998, 10, 93.

[13] T. Yamada, R. Kumai, Y. Takahashi, T. Hasegawa, J. Mater. Chem. 2010, 20, 5810.

[14] L. Jiang, H. Dong, W. Hu, J. Mater. Chem. 2010, 20, 4994.

[15] L. B. Coleman, M. J. Cohen, D. J. Sandman, F. G. Yamagishi, A. F. Garito, A. J. Heeger, Solid State Commun. 1973, 12, 1125.

[16] J. Ferraris, D. O. Cowan, V. Walatka, J. H. Perlstein, J. Am. Chem. Soc. 1973, 95, 948.

[17] J. M. Williams, J. R. Ferraro, R. J. Thorn, K. D. Carlson, U. Geiser, H. H. Wang, A. M. Kini, M. H. Wangbo, Organic Superconductors (Including Fullerenes) :

Synthesis, Structure, Properties, and Theory; Prentice Hall, Englewood Cliffs, New Jersey, 1992. 
[18] M. Mas-Torrent, C. Rovira, J. Mater. Chem. 2006, 16, 433.

[19] M. R. Bryce, Adv. Mater. 1999, 11, 11.

[20] J. Yamada, T. Sugimoto (Eds.), TTF Chemistry. Fundamentals and Applications of Tetrathiafulvalene; Kodansha Springer, 2004.

[21] H. Jiang, X. Yang, Z. Cui, Y. Liu, H. Li, W. Hu, C. Kloc, CrystEngComm 2014, 16, 5968.

[22] M. Mas-Torrent, C. Rovira, Chem. Soc. Rev. 2008, 37, 827.

[23] I. Doi, E. Miyazaki, K. Takimiya, Y. Kunugi, Chem. Mater. 2007, 19, 5230.

[24] X. Gao, W. Wu, Y. Liu, S. Jiao, W. Qiu, G. Yu, L. Wang, D. Zhu, J. Mater. Chem. 2007, 17, 736.

[25] X. Gao, W. Qiu, Y. Liu, G. Yu, D. Zhu, Pure Appl. Chem. 2008, 80, 2405.

[26] I. Doi, E. Miyazaki, K. Takimiya, Chem. Lett. 2008, 37, 1088.

[27] M. Katsuhara, I. Aoyagi, H. Nakajima, T. Mori, T. Kambayashi, M. Ofuji, Y. Takanishi, K. Ishikawa, H. Takezoe, H. Hosono, Synth. Met. 2005, 149, 219.

[28] Naraso, J. Nishida, D. Kumaki, S. Tokito, Y. Yamashita, J. Am. Chem. Soc. 2006, $128,9598$.

[29] B. Noda, M. Katsuhara, I. Aoyagi, T. Mori, T. Taguchi, T. Kambayashi, K. Ishikawa, H. Takezoe, Chem. Lett. 2005, 34, 392.

[30] S. Tamura, T. Kadoya, T. Kawamoto, T. Mori, Appl. Phys. Lett. 2013, 102, 063305.

[31] S. Tamura, T. Kadoya, T. Mori, Appl. Phys. Lett. 2014, 105, 023301.

[32] C.-C. Lee, S.-W. Liu, Y.-T. Chung, J. Phys. D: Appl. Phys. 2010, 43, 075102.

[33] J. Nishida, S. Ando, J. Yamaguchi, K. Itaka, H. Koinuma, H. Tada, S. Tokito, Y. Yamashita, J. Am. Chem. Soc. 2005, 127, 10142.

[34] B. Noda, M. Katsuhara, I. Aoyagi, H. Nakajima, T. Mori, T. Taguchi, T. Kambayashi, M. Ofuji, Y. Takanishi, K. Ishikawa, H. Takezoe, H. Hosono, Proc. Int. Symp. Super-Functionality Org. Devices 2004, 1, 154.

[35] T. Yamada, T. Hasegawa, M. Hiraoka, H. Matsui, Y. Tokura, G. Saito, Appl. Phys. Lett. 2008, 92, 233306.

[36] Naraso, J. Nishida, S. Ando, J. Yamaguchi, K. Itaka, H. Koinuma, H. Tada, S. Tokito, Y. Yamashita, J. Am. Chem. Soc. 2005, 127, 10142.

[37] R. A. L. Silva, A. I. Neves, M. L. Afonso, I. C. Santos, E. B. Lopes, F. Del Pozo, R. Pfattner, M. Mas-Torrent, C. Rovira, M. Almeida, D. Belo, Eur. J. Inorg. Chem. 2013, 2013, 2440.

[38] F. Otón, R. Pfattner, E. Pavlica, Y. Olivier, G. Bratina, J. Cornil, J. Puigdollers, R. Alcubilla, X. Fontrodona, M. Mas-Torrent, J. Veciana, C. Rovira, CrystEngComm 2011, 13, 6597.

[39] T. Takahashi, S. Tamura, Y. Akiyama, T. Kadoya, T. Kawamoto, T. Mori, Appl. Phys. Express 2012, 5, 061601.

[40] J. Nagakubo, M. Ashizawa, T. Kawamoto, A. Tanioka, T. Mori, Phys. Chem. Chem. Phys. 2011, 13, 14370.

[41] B. Noda, H. Wada, K. Shibata, T. Yoshino, M. Katsuhara, I. Aoyagi, T. Mori, T. Taguchi, T. Kambayashi, K. Ishikawa, H. Takezoe, Nanotechnology 2007, 18, 424009.

[42] K. Shibata, K. Ishikawa, H. Takezoe, H. Wada, T. Mori, Appl. Phys. Lett. 2008, 92, 023305.

[43] J. Inoue, M. Kanno, M. Ashizawa, C. Seo, A. Tanioka, T. Mori, Chem. Lett. 2010, 39, 538.

[44] X. K. Gao, Y. Wang, X. D. Yang, Y. Q. Liu, W. F. Qiu, W. P. Wu, H. J. Zhang, T. Qi, Y. Liu, K. Lu, C. Y. Du, Z. G. Shuai, G. Yu, D. B. Zhu, Adv. Mater. 2007, 19, 3037. 
[45] K. Omata, M. Mamada, J. Nishida, S. Tokito, Y. Yamashita, Bull. Chem. Soc. Jpn. 2010, 83, 575.

[46] G. Yang, C. Di, G. Zhang, J. Zhang, J. Xiang, D. Zhang, D. Zhu, Adv. Funct. Mater. 2013, 23, 1671.

[47] M. Kanno, Y. Bando, T. Shirahata, J. Inoue, H. Wada, T. Mori, J. Mater. Chem. 2009, 19, 6548.

[48] M.-S. Nam, a. Ardavan, R. J. Cava, P. M. Chaikin, Appl. Phys. Lett. 2003, 83, 4782.

[49] H. Xie, H. Alves, A. F. Morpurgo, Phys. Rev. B 2009, 80, 245305.

[50] Y. Takahashi, T. Hasegawa, S. Horiuchi, R. Kumai, Y. Tokura, G. Saito, Chem. Mater. 2007, 19, 6382.

[51] A. Tracz, T. Pakula, J. K. Jeszka, Mater. Sci.-Pol. 2004, 22, 415.

[52] A. Tracz, J. Ulanski, T. Pakula, M. Kryszewski, P-131986 1986.

[53] S. Kotarba, J. Jung, A. Kowalska, T. Marszalek, M. Kozanecki, P. Miskiewicz, M. Mas-Torrent, C. Rovira, J. Veciana, J. Puigmarti-Luis, J. Ulanski, J. Appl. Phys. 2010, 108, 014504.

[54] M. Mas-Torrent, S. Masirek, P. Hadley, N. Crivillers, N. S. Oxtoby, P. Reuter, J. Veciana, C. Rovira, a. Tracz, Org. Electron. 2008, 9, 143.

[55] X. Gao, W. Wu, Y. Liu, W. Qiu, X. Sun, G. Yu, D. Zhu, Chem. Commun. 2006, 2750 .

[56] L. Tan, Y. Guo, Y. Yang, G. Zhang, D. Zhang, G. Yu, W. Xu, Y. Liu, Chem. Sci. 2012, 3, 2530.

[57] I. a. Wright, N. J. Findlay, S. Arumugam, A. R. Inigo, A. L. Kanibolotsky, P. Zassowski, W. Domagala, P. J. Skabara, J. Mater. Chem. C 2014, 2, 2674.

[58] T. Yoshino, K. Shibata, H. Wada, Y. Bando, K. Ishikawa, H. Takezoe, T. Mori, Chem. Lett. 2009, 38, 200.

[59] H. Jiang, X. Yang, Z. Cui, Y. Liu, H. Li, W. Hu, Y. Liu, D. Zhu, Appl. Phys. Lett. 2007, 91, 123505.

[60] H. Jiang, X. Yang, E. Wang, Y. Fu, Y. Liu, H. Li, Z. Cui, Y. Liu, W. Hu, Synth. Met. 2011, 161, 136.

[61] M. Leufgen, O. Rost, C. Gould, G. Schmidt, J. Geurts, L. W. Molenkamp, N. S. Oxtoby, M. Mas-Torrent, N. Crivillers, J. Veciana, C. Rovira, Org. Electron. 2008, 9, 1101.

[62] M. Mas-Torrent, P. Hadley, S. T. Bromley, N. Crivillers, J. Veciana, C. Rovira, Appl. Phys. Lett. 2005, 86, 012110.

[63] M. Mas-torrent, P. Hadley, S. T. Bromley, X. Ribas, J. Tarre, M. Mas, E. Molins, J. Veciana, C. Rovira, J. Am. Chem. Soc. 2004, 126, 8546.

[64] M. Mas-Torrent, P. Hadley, X. Ribas, C. Rovira, Synth. Met. 2004, 146, 265.

[65] R. Pfattner, M. Mas-Torrent, I. Bilotti, A. Brillante, S. Milita, F. Liscio, F. Biscarini, T. Marszalek, J. Ulanski, A. Nosal, M. Gazicki-Lipman, M. Leufgen, G. Schmidt, L. W. Molenkamp, V. Laukhin, J. Veciana, C. Rovira, Adv. Mater. 2010, 22, 4198.

[66] R. Pfattner, M. Mas-Torrent, C. Moreno, J. Puigdollers, R. Alcubilla, I. Bilotti, E. Venuti, A. Brillante, V. Laukhin, J. Veciana, C. Rovira, J. Mater. Chem. 2012, 22, 16011.

[67] Y. Liu, Y. Han, X. Zhao, Y. Tong, Q. Tang, Y. Liu, Synth. Met. 2014, 198, 248.

[68] Y. Y. Liu, X. Zhao, B. Cai, T. Pei, Y. Tong, Q. Tang, Y. Y. Liu, Nanoscale 2014, 6, 1323.

[69] N. M. Tucker, A. L. Briseno, O. Acton, H. Yip, H. Ma, S. A. Jenekhe, Y. Xia, A. K. Jen, Appl. Mater. Interfaces 2013, 5, 2320.

[70] T. Marszalek, a. Nosal, R. Pfattner, J. Jung, S. Kotarba, M. Mas-Torrent, B. Krause, J. Veciana, M. Gazicki-Lipman, C. Crickert, G. Schmidt, C. Rovira, J. Ulanski, Org. Electron. 2012, 13, 121. 
[71] S. Ukai, S. Igarashi, M. Nakajima, K. Marumoto, H. Ito, S. Kuroda, K. Nishimura, Y. Enomoto, G. Saito, Colloids Surf., A 2006, 284-285, 589.

[72] H. Inokuchi, G. Saito, P. Wu, K. Seki, T. B. Tang, T. Mori, K. Imaeda, T. Enoki, Y. Higuchi, K. Inaka, N. Yasuoka, Chem. Lett. 1986, 15, 1263.

[73] Y. Geng, R. Pfattner, A. Campos, J. Hauser, V. Laukhin, J. Puigdollers, J. Veciana, M. Mas-Torrent, C. Rovira, S. Decurtins, S.-X. Liu, Chem. - Eur. J. 2014, 20, 7136.

[74] Y. Geng, R. Pfattner, A. Campos, W. Wang, O. Jeannin, J. Hauser, J. Puigdollers, S. T. Bromley, S. Decurtins, J. Veciana, C. Rovira, M. Mas-Torrent, S.-X. Liu, Chem. Eur. J. 2014, 20, 16672.

[75] M. Mas-Torrent, C. Rovira, Chem. Rev. 2011, 111, 4833.

[76] L. Antolini, G. Horowitz, F. Kouki, F. Garnier, Adv. Mater. 1998, 10, 382.

[77] A. Brillante, I. Bilotti, F. Biscarini, R. G. Della Valle, E. Venuti, Chem. Phys. 2006, 328, 125.

[78] P. A. Chaloner, S. R. Gunatunga, P. B. Hitchcock, Acta Crystallogr. C 1994, 50, 1941.

[79] G. Horowitz, B. Bachet, A. Yassar, P. Lang, F. Demanze, J.-L. Fave, F. Garnier, Chem. Mater. 1995, 7, 1337.

[80] M. Pelletier, F. Brisse, Acta Crystallogr. C 1994, 50, 1942.

[81] W. Porzio, S. Destri, M. Mascherpa, S. Rossini, S. Brückner, Synth. Met. 1993, 55, 408.

[82] P. Ranzieri, A. Girlando, S. Tavazzi, M. Campione, L. Raimondo, I. Bilotti, A. Brillante, R. G. Della Valle, E. Venuti, ChemPhysChem 2009, 10, 657.

[83] D. Schneider, T. Rabe, T. Riedl, T. Dobbertin, M. KrÃ Iger, E. Becker, H.-H. Johannes, W. Kowalsky, T. Weimann, J. Wang, P. Hinze, A. Gerhard, P. StÃ $q s s e l, ~ H$. Vestweber, Adv. Mater. 2005, 17, 31.

[84] T. Siegrist, C. Kloc, J. H. Schön, B. Batlogg, R. C. Haddon, S. Berg, G. A. Thomas, Angew. Chem. 2001, 40, 1732.

[85] A. Brillante, R. G. Della Valle, L. Farina, A. Girlando, M. Masino, E. Venuti, Chem. Phys. Lett. 2002, 357, 32.

[86] R. B. Campbell, J. M. Robertson, J. Trotter, Acta Crystallogr. 1961, 14, 705.

[87] R. G. Della Valle, E. Venuti, A. Brillante, A. Girlando, J. Chem. Phys. 2003, 118, 807.

[88] L. Farina, A. Brillante, R. G. Della Valle, E. Venuti, M. Amboage, K. Syassen, Chem. Phys. Lett. 2003, 375, 490.

[89] D. Holmes, S. Kumaraswamy, A. J. Matzger, K. P. C. Vollhardt, Chem. - Eur. J. 1999, 5, 3399.

[90] T. Kakudate, N. Yoshimoto, Y. Saito, Appl. Phys. Lett. 2007, 90, 081903.

[91] D. H. Kim, H. S. Lee, H. Yang, L. Yang, K. Cho, Adv. Funct. Mater. 2008, 18, 1363.

[92] C. C. Mattheus, A. B. Dros, J. Baas, A. Meetsma, J. L. de Boer, T. T. M. Palstra, Acta Crystallogr. C 2001, 57, 939.

[93] C. C. Mattheus, A. B. Dros, J. Baas, G. T. Oostergetel, A. Meetsma, J. L. de Boer, T. T. M. Palstra, Synth. Met. 2003, 138, 475.

[94] T. Siegrist, C. Kloc, R. A. Laudise, H. E. Katz, R. C. Haddon, Adv. Mater. 1998, 10, 379.

[95] G. J. Visser, G. J. Heeres, J. Wolters, A. Vos, Acta Crystallogr. B 1968, 24, 467.

[96] Y. Diao, K. M. Lenn, W. Lee, M. A. Blood-Forsythe, J. Xu, Y. Mao, Y. Kim, J. A. Reinspach, S. Park, G. Xue, P. Clancy, A. Aspuru-Guzik, G. Xue, P. Clancy, Z. Bao, S. C. B. Mannsfeld, J. Am. Chem. Soc. 2014, 136, 17046.

[97] E. Gann, X. Gao, C. Di, C. R. McNeill, Adv. Funct. Mater. 2014, 24, 7211. 
[98] G. Giri, R. Li, D.-M. Smilgies, E. Q. Li, Y. Diao, K. M. Lenn, M. Chiu, D. W. Lin, R. Allen, J. Reinspach, S. C. B. Mannsfeld, S. T. Thoroddsen, P. Clancy, Z. Bao, A. Amassian, Nat. Commun. 2014, 5, 3573.

[99] T. He, M. Stolte, C. Burschka, N. H. Hansen, T. Musiol, D. Kälblein, J. Pflaum, X. Tao, J. Brill, F. Würthner, Nat. Commun. 2015, 6, 5954.

[100] A. M. Hiszpanski, R. M. Baur, B. Kim, N. J. Tremblay, C. Nuckolls, A. R. Woll, Y. Loo, J. Am. Chem. Soc. 2014, 136, 15749.

[101] O. D. Jurchescu, D. A. Mourey, S. Subramanian, S. R. Parkin, B. M. Vogel, J. E. Anthony, T. N. Jackson, D. J. Gundlach, Phys. Rev. B 2009, 80, 085201.

[102] W. F. Cooper, N. C. Kenny, J. W. Edmonds, a. Nagel, F. Wudl, P. Coppens, J. Chem. Soc. D 1971, 889.

[103] A. Ellern, J. Bernstein, J. Y. Becker, S. Zamir, L. Shahal, S. Cohen, Chem. Mater. 1994, 6, 1378.

[104] R. Pfattner, C. Moreno, C. Voz, R. Alcubilla, C. Rovira, J. Puigdollers, M. MasTorrent, Org. Electron. 2014, 15, 211.

[105] C. Rovira, J. Veciana, N. Santalo, J. Tarres, J. Cirujeda, E. Molins, J. Llorca, E. Espinosa, J. Org. Chem. 1994, 59, 3307.

[106] A. Brillante, I. Bilotti, R. G. Della Valle, E. Venuti, M. Mas-Torrent, C. Rovira, Y. Yamashita, Chem. Phys. Lett. 2012, 523, 74.

[107] A. Brillante, I. Bilotti, R. G. Della Valle, E. Venuti, S. Milita, C. Dionigi, F. Borgatti, A. N. Lazar, F. Biscarini, M. Mas-Torrent, N. S. Oxtoby, N. Crivillers, J. Veciana, C. Rovira, M. Leufgen, G. Schmidt, L. W. Molenkamp, CrystEngComm 2008, 10, 1899.

[108] T. Terauchi, S. Sumi, Y. Kobayashi, Y. Matsushita, A. Sato, Cryst. Growth Des. 2014, 14, 1412.

[109] Q. Wang, J. D. Wallis, Y. Wu, M. Pilkington, CrystEngComm 2014, 16, 10235.

[110] L. Duan, J. Qiao, Y. Sun, Y. Qiu, Adv. Mater. 2011, 23, 1137.

[111] F. Otón, R. Pfattner, E. Pavlica, Y. Olivier, E. Moreno, J. Puigdollers, G. Bratina, J. Cornil, X. Fontrodona, M. Mas-Torrent, J. Veciana, C. Rovira, Chem. Mater. 2011, 23, 851.

[112] A. R. Murphy, J. M. J. Fréchet, Chem. Rev. 2007, 107, 1066.

[113] A. Amacher, H. Luo, Z. Liu, M. Bircher, M. Cascella, J. Hauser, S. Decurtins, D. Zhang, S.-X. Liu, RSC Adv. 2014, 4, 2873.

[114] R. Pfattner, E. Pavlica, M. Jaggi, S.-X. Liu, S. Decurtins, G. Bratina, J. Veciana, M. Mas-Torrent, C. Rovira, J. Mater. Chem. C 2013, 1, 3985.

[115] N. Borjigin, J. Nishida, S. Tokito, L. Theogarajan, Y. Yamashita, Synth. Met. 2010, 160, 2323.

[116] J. Cornil, J.-L. Brédas, J. Zaumseil, H. Sirringhaus, Adv. Mater. 2007, 19, 1791.

[117] E. J. Meijer, D. M. de Leeuw, S. Setayesh, E. van Veenendaal, B. H. Huisman, P. W. M. Blom, J. C. Hummelen, U. Scherf, T. M. Klapwijk, Nat. Mater. 2003, 2, 678.

[118] S. T. Bromley, F. Illas, M. Mas-Torrent, Phys. Chem. Chem. Phys. 2008, 10, 121.

[119] C. Moreno, R. Pfattner, M. Mas-Torrent, J. Puigdollers, S. T. Bromley, C. Rovira, J. Veciana, R. Alcubilla, J. Mater. Chem. 2012, 22, 345.

[120] R. A. Marcus, Rev. Mod. Phys. 1993, 65, 599.

[121] J.-L. Brédas, D. Beljonne, V. Coropceanu, J. Cornil, Chem. Rev. 2004, 104, 4971.

[122] F. Otón, R. Pfattner, N. S. Oxtoby, M. Mas-Torrent, K. Wurst, X. Fontrodona, Y. Olivier, J. Cornil, J. Veciana, C. Rovira, J. Org. Chem. 2011, 76, 154.

[123] H. Li, R. Zheng, Q. Shi, Phys. Chem. Chem. Phys. 2011, 13, 5642.

[124] G. Nan, Z. Li, Org. Electron. 2012, 13, 1229.

[125] A. Troisi, G. Orlandi, J. Phys. Chem. A 2006, 110, 4065. 
[126] J.-F. Chang, T. Sakanoue, Y. Olivier, T. Uemura, M.-B. Dufourg-Madec, S. G. Yeates, J. Cornil, J. Takeya, A. Troisi, H. Sirringhaus, Phys. Rev. Lett. 2011, 107, 066601.

[127] K. Marumoto, S. Kuroda, T. Takenobu, Y. Iwasa, Phys. Rev. Lett. 2006, 97, 256603.

[128] E. Demiralp, W. A. Goddard, J. Phys. Chem. A 1997, 101, 8128.

[129] S. T. Bromley, M. Mas-torrent, P. Hadley, C. Rovira, J. Am. Chem. Soc. 2004, 126, 6544.

[130] J. Casado, M. Z. Zgierski, M. Carmen, R. Delgado, J. T. L. Navarrete, M. Mastorrent, C. Rovira, J. Phys. Chem. C 2007, 111, 10110.

[131] H. Li, X. Wang, Z. Li, Chin. Sci. Bull. 2012, 57, 4049.

[132] H. Li, R. Zheng, Q. Shi, J. Phys. Chem. C 2012, 116, 11886.

[133] N. E. Gruhn, D. A. da Silva Filho, T. G. Bill, M. Malagoli, V. Coropceanu, A. Kahn, J.-L. Brédas, J. Am. Chem. Soc. 2002, 124, 7918.

[134] H. Li, X. Wang, Z. Li, R. Zheng, Y. Zhu, Sci. China Chem. 2012, 55, 2176.

[135] V. Lemaur, D. A. da Silva Filho, V. Coropceanu, M. Lehmann, Y. Geerts, J. Piris, M. G. Debije, A. M. van de Craats, K. Senthilkumar, L. D. A. Siebbeles, J. M. Warman, J.-L. Brédas, J. Cornil, J. Am. Chem. Soc. 2004, 126, 3271.

[136] J. Torras, S. Bromley, O. Bertran, F. Illas, Chem. Phys. Lett. 2008, 457, 154.

[137] W. Zhang, W. Liang, Y. Zhao, J. Chem. Phys. 2010, 133, 024501.

[138] R. Pfattner, C. Rovira, M. Mas-Torrent, Phys. Chem. Chem. Phys. 2014.

[139] T. Kadoya, S. Tamura, T. Mori, J. Phys. Chem. C 2014, 118, 23139.

[140] C. Di, F. Zhang, D. Zhu, Adv. Mater. 2013, 25, 313.

[141] T. Kakinuma, H. Kojima, T. Kawamoto, T. Mori, J. Mater. Chem. C 2013, 1, 2900.

[142] M. Mas-Torrent, P. Hadley, N. Crivillers, J. Veciana, C. Rovira, ChemPhysChem 2006, 7, 86.

[143] A. Nollau, M. Pfeiffer, T. Fritz, K. Leo, J. Appl. Phys. 2000, 87, 4340.

[144] Z. Wei, H. Xi, H. Dong, L. Wang, W. Xu, W. Hu, D. Zhu, J. Mater. Chem. 2010, 20, 1203.

[145] K. P. Goetz, D. Vermeulen, M. E. Payne, C. Kloc, L. E. McNeil, O. D. Jurchescu, J. Mater. Chem. C 2014, 2, 3065. 